\title{
Influence of evaporation on the morphology of a thin film of a partially miscible binary mixture
}

\author{
Ramin Rabani ${ }^{\mathrm{a}, *}$, Hosein Sadafi ${ }^{\mathrm{b}}$, Hatim Machrafi ${ }^{\mathrm{a}}$,Monavar Abbasi ${ }^{\mathrm{a}}$, Benoit \\ Haut $^{\mathrm{b}}$, Pierre Dauby ${ }^{\mathrm{a}}$ \\ ${ }^{\mathrm{a}}$ University of Liège, Thermodynamics of Irreversible Phenomena, Allée du 6-Août 19, 4000 \\ Liège, Belgium \\ ${ }^{b}$ Université libre de Bruxelles, TIPs Laboratory Avenue F.D. Roosevelt 50, CP165/67, 1050 \\ Bruxelles, Belgium \\ *Corresponding author: ramin.rabani@uliege.be \\ mhsadafi@gmail.com, H.Machrafi@uliege.be, monavar.abbasi@gmail.com, bhaut@ulb.ac.be, \\ PC.Dauby@uliege.be
}

\begin{abstract}
In this paper, we develop numerical, theoretical and experimental analyses of the different morphologies that can be created by the phase separation phenomena that are induced by solvent evaporation in a thin film of a partially miscible binary mixture. Disregarding hydrodynamic effects, the Cahn-Hilliard-Cook and temperature equations are used to describe the thermodynamics of non-isothermal phase separation in a 2D thin film. Numerical simulations are performed to investigate the interplay between evaporation and phase separation and we examine the effect on the morphology of the film of several parameters such as the initial thickness of the layer, or the initial temperature and concentration of the mixture. Interestingly, the competition between evaporation and phase separation is shown to be the main determinant of the choice between a lamellar or a lateral pattern in the beginning of phase separation. For moderate evaporation rate, the spinodal instability takes place close to the evaporating interface and a lateral structure is formed. For stronger evaporation, the spinodal instability does not occur and a lamellar structure is created. In addition, the mid- or long- term evolution of the system is also considered. The thickness of the film is an important parameter in this analysis and possible modifications of the pattern over time are emphasized. Detailed physical and theoretical interpretations are proposed for the results and experiments in a Hele-Shaw cell that nicely confirm our predictions are presented.
\end{abstract}

Keywords: thin film, partially miscible binary mixture, evaporation, phase separation morphology, lateral and lamellar structures, coalescence 


\section{Introduction}

\subsection{Physical concept of phase separation}

Phase separation takes place in many industrial processes related to semiconductors $[1,2]$, binary alloys $[3,4]$, polymer blends $[5,6], \ldots$ For a binary mixture of partially miscible liquids consisting of a solvent and a solute, demixing is associated with a "miscibility gap" in the phase diagram of the mixture (see Fig.1). This phase diagram has three regions: the stable (mixed), metastable, and unstable (demixed) ones. The phase diagram is defined by two curves: the binodal and the spinodal curves. The binodal curve defines the boundary between the stable and the metastable regions while the spinodal curve is the boundary between the metastable and the unstable regions $[7,8]$. When the representative point of a mixture crosses one of these curves and enters either in the metastable or in the unstable region, phase separation occurs. It means that the solution separates to create two phases of different compositions. In the metastable region, phase separation occurs through nucleation and growth, while spinodal decomposition happens in the unstable region $[7,8]$. When a mixture is suddenly quenched below the spinodal curve, the phase separation mechanism may be divided into the following three stages: (a) early stage, (b) intermediate stage, and (c) late stage [9-11]. In the early stage of the spinodal decomposition, the concentration fluctuations are small and weakly nonlinear. Therefore, the characteristic wavelength of the domain's size does not change with time while the amplitude of the concentration fluctuations increases with time. The phase separation at this stage can be described by the linearized Cahn-Hilliard equation [9-13]. In the intermediate stage, the concentration fluctuations and the characteristic wavelength both increase with time. The nonlinear effects on the time evolution of the concentration fluctuations become increasingly important in this stage. As a consequence, growth of the concentration fluctuations is governed by a nonlinear time evolution equation [9-11]. In the late stage, the equilibrium concentrations are reached and the domain size is coarsening without a change in concentration, whereas the wavelength increases with time due to coarsening [9-11].

The kinetics of phase separation by demixing has been the focus of many theoretical and experimental studies [14-17]. In the absence of surface effects, the phase separation of a binary mixture in a bulk can typically generate two types of morphologies according to the average concentration. If the concentration of the binary mixture is not the critical concentration (i.e. if the solute volume fraction $\emptyset_{\mathrm{sl}} \neq 0.5$, see Fig. 1), a droplet-type morphology forms [18-20]. On the contrary, an interconnected-type morphology forms for the critical concentration [18-20]. The presence of a surface may also alter the morphology type and preferential wetting of the surface by one of the components becomes a relevant parameter [21-23]. 


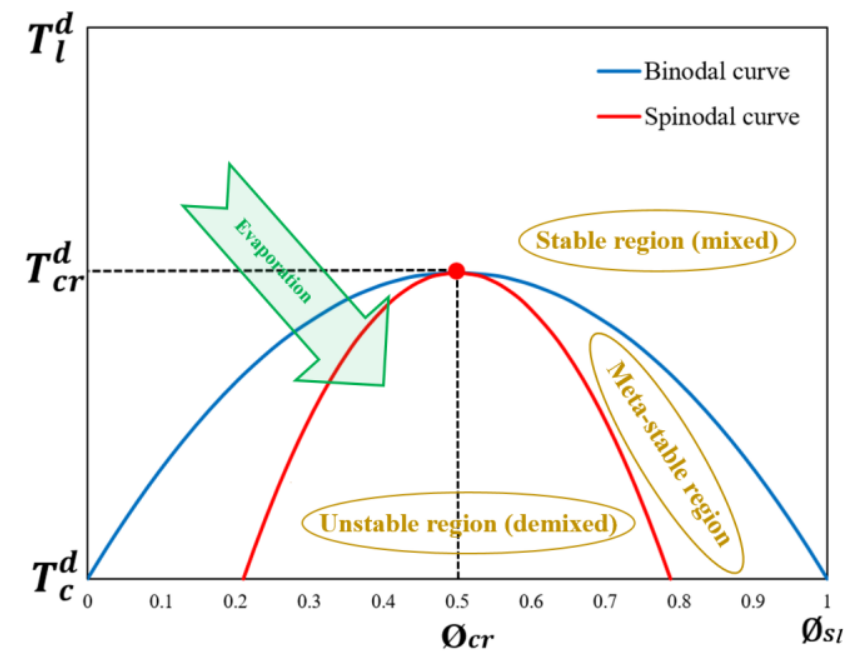

Fig. 1. Binodal and spinodal curves in a phase diagram. $\emptyset_{\mathrm{sl}}$ is the solute volume fraction. $T_{l}^{d}$, $T_{c r}^{d}$, and $T_{c}^{d}$ are respectively the liquid phase temperature, the critical temperature, and the minimum temperature, which corresponds to $\emptyset_{\mathrm{sl}}=0$ and $\emptyset_{\mathrm{sl}}=1$ in the binodal curve.

Both temperature and concentration can be changed in the phase diagram to induce phase separation (see Fig. 1). Phase separation induced by a decrease in temperature is known as thermally-induced phase separation and is usually performed by cooling down the binary mixture, while the mean concentration of the mixture remains constant [24-26]. Phase separation can also be induced by a change in the concentrations of the different components of a mixture. This process can be performed by directly adding some components in the mixture [27, 28]. Evaporation can also induce concentration changes in the system, and this phenomenon is the object of the present paper. Note however that in this situation, temperature changes are also expected because evaporation induces a cooling of the system.

\subsection{Phase separation in a thin evaporating film}

The evaporation of thin films has attracted many interests because it commonly happens in natural phenomena and in many industrial processes such as drying of paint films [29-31] ink-jet printing [32-34], packaging [35, 36], heat exchangers [37, $38]$, and fabrication of solar cells [39, 40]. When a thin film consists of a binary mixture of partially miscible liquids, either a temperature gradient [41], or a concentration gradient [42], or both as in the case of evaporation [43], can induce demixing. The situation of evaporation, which is analyzed in the present paper, provides an example of phase separation induced by phase change. The interplay between surface and bulk behavior for the phase separation in a thin film can lead to different types of morphologies, such as the lateral and lamellar structures [44-46]. The lamellar structure is characterized by a horizontal homogeneity and the separated phases form superposed layers within the film. On the contrary, the lateral structure consists of arrangements of columns, or vertical stripes of the separated 
components. These morphologies occur when altering the system characteristics such as the composition [47], or changes in the external conditions including the wetting behavior on the surfaces [48] and the temperature [24]. These conditions directly control the formation and the evolution of the demixing process and thus create the different types of morphologies.

Evaporation induced phase separation in a partially miscible mixture has received rather little attention so far in the literature. Zoumpouli et al. [49] performed a numerical analysis of the phase separation morphology developed in a thin film containing a volatile solvent and two dissolved polymers. They modeled the evaporation by a purely empirical model. They observed that, for a system with preferential wetting, diffusion alone favors a lamellar structure for the separated phases in the film. However, hydrodynamic effects may deform and transform the lamellar structure to a lateral structure. Negi et al. [50] developed three-dimensional simulations of evaporation-induced phase separation in a polymer solution. They observed that phase separation for fast evaporation rates proceeds in an inhomogeneous manner, with droplets appearing first at the top of the film. For a slow evaporation rate, the solvent has enough time to diffuse from the bulk and, thus, phase separation occurs uniformly in the bulk. Another analysis of an evaporating and demixing fluid layer is provided by Cummings et al. [51]. In this study, a thermodynamic deduction of the equations is proposed for a mixture of two polymers and a solvent, but the system is assumed isothermal. Some ad hoc coefficients are also introduced to describe evaporation. Dayal and Kyu [52] examined numerically the competition between the dynamics of phase separation and the rate of solvent evaporation and its effect on the morphology of a single polymer-solvent in a cylindrical geometry. They observed a skin layer of polymer at the outer surface of the cylindrical fiber for fast evaporation, while a porous structure was obtained for slow evaporation. Another study of an evaporating and isothermal phase separation for a binary mixture consisting of a single polymer and solvent is provided by Schaefer et al. [53]. In this study, the authors examined theoretically and numerically the impact of the solvent evaporation on the dynamics of the isothermal phase separation of a binary mixture in order to explain how solvent evaporation affects the appearance and development of structural length scales. They solved their model only in the limit of small Biot numbers (expressing the ratio between the rate of external mass transport by evaporation and internal mass transport by diffusion), where the system is uniform along the height of the thin film. In the early stages of demixing, they observed a spinodal length scale that decreases with time under the influence of the solvent evaporation. They also achieved a good agreement between the theoretical model and numerical simulations for the early stages of demixing. 
According to our knowledge and to the literature review presented above, demixing in the evaporation process of a thin film of a partially miscible binary mixture has been mainly studied in the case of an isothermal situation. There is limited information in the literature on non-isothermal demixing induced by evaporation, in particular on the path to the unstable zone in the phase diagram. In our previous work [43], we analyzed experimentally and theoretically the evaporation induced demixing in sessile drops of binary mixtures of partially miscible liquids. We proposed a simple model with the aim to analyze the influence of the initial conditions on the possible onset of demixing (but we did not model the demixing phase separation itself). Notably, we showed that demixing can be induced even if the initial temperature of the system is above the critical temperature, due to the cooling generated by its evaporation.

\subsection{Objective of this work}

The main purpose of the present study is to analyse the demixing phenomenon induced by the evaporation of a volatile solvent in a two-dimensional thin film of a partially miscible binary mixture consisting of a solvent and a solute (the solute is assumed nonvolatile). The model is based on a non-isothermal phase field approach to describe the behavior of phase separation in a multicomponent system. Hydrodynamics effects and convective flow are not modeled in the present paper and will be considered in a future study. Therefore, we focus solely on the diffusive dynamics and the morphology evolution in the system. We present and solve a numerical model based on the Cahn-Hilliard-Cook and heat transfer equations. We perform numerical simulations to examine the competition between the evaporation and the phase separation and we analyze the role of the initial temperature, the initial solvent concentration, and the initial thickness of the film. Additionally, we describe an experimental set-up that has been used to show that the trends regarding the morphology highlighted by the numerical simulations can also be observed experimentally.

\section{Methods and model formulation}

In this section, we describe the phase field model and the heat transfer equation used to simulate the morphology of phase separation during the evaporation of a thin film of a partially miscible binary mixture of solvent-solute, of which solely the solvent evaporates into an inert gas, air (Fig. 2). As discussed in the introduction, convection is disregarded in the present study. A 2D rectangular domain is considered, with $x$ the horizontal coordinate and $z$ the vertical one. 


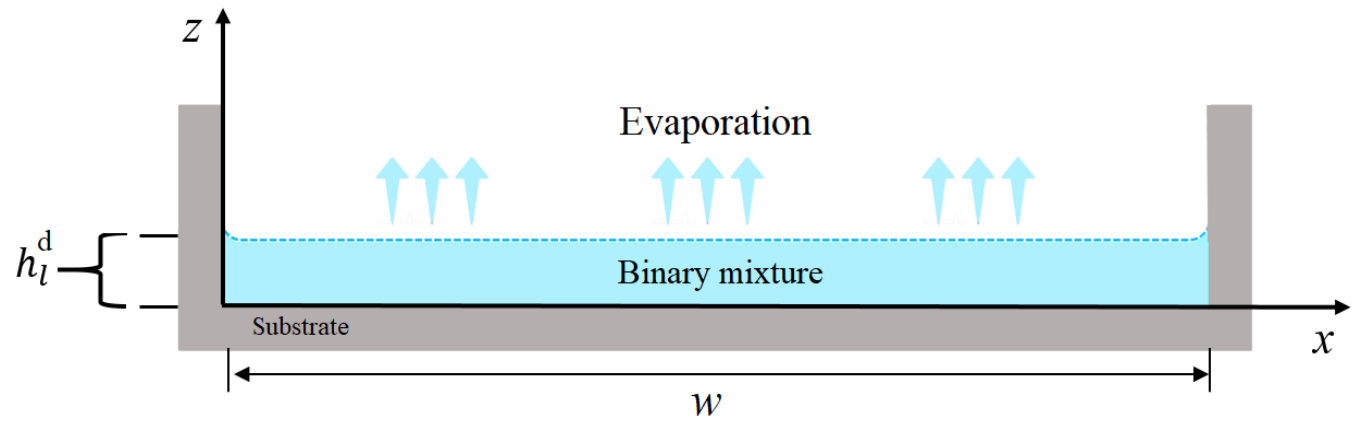

Fig. 2. Schematic of the studied configuration.

\subsection{Order parameters}

The local composition of the binary mixture can be described by the mass fractions of the components $C_{\mathrm{sv}}$ and $C_{\mathrm{sl}}\left(C_{\mathrm{sl}}=1-C_{\mathrm{sv}}\right)$. The subscripts "sv" and "sl" stand for the solvent and the solute, respectively. The local composition can also be expressed in terms of the solvent volume fraction $\emptyset_{\mathrm{sv}}$ with

$$
C_{\mathrm{sv}}=\frac{\rho_{\mathrm{sv}} \emptyset_{\mathrm{sv}}}{\rho_{\mathrm{sv}} \emptyset_{\mathrm{sv}}+\rho_{\mathrm{sl}}\left(1-\emptyset_{\mathrm{sv}}\right)}
$$

where $\rho_{\mathrm{sv}}$ and $\rho_{\mathrm{sl}}$ are the densities of the solvent and the solute, respectively. Since, in our system, we assume that the densities of solvent and solute are not too different, $C_{\mathrm{sv}}$ and $\emptyset_{\mathrm{sv}}$ are almost identical. Therefore, all equations are presented in this paper in terms of the volume fraction $\emptyset_{\mathrm{sv}}$.

\subsection{Free energy and governing equations}

General theories on phase field models have been developed in several papers (see for instance [54-57]) and we recall here the basic ingredients only. The free energy density $F$ of a non-isothermal mixture of two fluids can be written in the GinzburgLandau form:

$$
F\left(\emptyset_{\mathrm{sv}}, \nabla \emptyset_{\mathrm{sv}}, T_{l}^{d}\right)=f_{0}\left(\emptyset_{\mathrm{sv}}, T_{l}^{d}\right)+\frac{1}{2} \varepsilon^{2}\left|\nabla \emptyset_{\mathrm{sv}}\right|^{2}
$$

with $T_{l}^{d}$ the local liquid phase temperature. Note that the superscript " $d$ " indicates that the quantity is dimensional. The first term in Eq. (2) is called the potential function and it depends on the local concentration and temperature. The interfacial energy, described by the second term in Eq. (2), depends on the concentration gradient and $\varepsilon$, the capillary width characterizing the thickness of the diffuse interface (interfacial thickness) between the two phases [58-60]. It is worth 
mentioning that the interfacial thickness may range from $10^{-9} \mathrm{~m}$ up to $10^{-7} \mathrm{~m}$ for two partially miscible substances [61-63].

We need a double-well potential function in order to describe the possible separation of the binary mixture into two distinct phases. For convenience, we use the simplest way to describe this double-well potential function and introduce a fourth order polynomial with respect to $\emptyset_{\mathrm{sv}}$, taking the following form $[64,65]$ :

$$
f_{0}\left(\emptyset_{\mathrm{sv}}, T_{l}^{d}\right)=\frac{1}{4}\left(\left(2 \emptyset_{\mathrm{sv}}-1\right)^{2}+\left(\frac{T_{l}^{d}-T_{c r}^{d}}{T_{c r}^{d}-T_{c}^{d}}\right)\right)^{2}
$$

where $T_{c r}^{d}$, and $T_{c}^{d}$ are the critical and the minimum temperature, respectively (see Fig. 1). The minima of the double-well potential function determine the concentrations of the separated phases at equilibrium, as functions of the temperature, and, subsequently, the binodal curve (see Fig. 1). In addition, the spinodal curve can be built by plotting, as a function of the temperature, the inflection points of the double-well potential function (see Fig. 1).

Based on Eq. (2), the governing equations can be formulated. A generalized chemical potential for a binary mixture can be defined: $\mu=\delta F / \delta \emptyset_{\mathrm{sv}}$, where $\delta / \delta \emptyset_{\mathrm{sv}}$ is the functional derivative. Using the continuity equation $\frac{\partial \emptyset_{\mathrm{sv}}}{\partial t}=-\vec{\nabla} \cdot \vec{J}$ and Fick's law for the mass flux $\vec{J}=-M \vec{\nabla} \mu$, where $M$ is the mobility, which is assumed constant, the Cahn-Hilliard equation is obtained:

$$
\begin{aligned}
\frac{\partial \emptyset_{\mathrm{sv}}}{\partial t} & =M \nabla^{2} \mu \\
& =M \nabla^{2}\left(-\varepsilon^{2} \nabla^{2} \emptyset_{\mathrm{sv}}+2\left(2 \emptyset_{\mathrm{sv}}-1\right)\left(\left(2 \emptyset_{\mathrm{sv}}-1\right)^{2}+\left(\frac{T_{l}^{d}-T_{c r}^{d}}{T_{c r}^{d}-T_{c}^{d}}\right)\right)\right)
\end{aligned}
$$

To introduce a possible thermal noise $\xi_{\mathrm{s}}$ in the description, an additional term is added to the equation above and one obtains the Cahn-Hilliard-Cook equation [66]:

$$
\frac{\partial \emptyset_{\mathrm{sv}}}{\partial t}=M \nabla^{2}\left(-\varepsilon^{2} \nabla^{2} \emptyset_{\mathrm{sv}}+2\left(2 \emptyset_{\mathrm{sv}}-1\right)\left(\left(2 \emptyset_{\mathrm{sv}}-1\right)^{2}+\left(\frac{T_{l}^{d}-T_{c r}^{d}}{T_{c r}^{d}-T_{c}^{d}}\right)\right)\right)+\sigma \xi_{\mathrm{s}}
$$

The thermal noise $\xi_{\mathrm{s}}$ is assumed to obey the fluctuation-dissipation theorem, i.e. $\left\langle\xi_{\mathrm{s}}(r, t)\right\rangle=0$, and $\left\langle\xi_{\mathrm{s}}(r, t) \xi_{\mathrm{s}}\left(r^{\prime}, t^{\prime}\right)\right\rangle=-M \nabla^{2} \delta\left(r-r^{\prime}\right) \delta\left(t-t^{\prime}\right) \quad[66$, 67]. The dimensionless parameter $\sigma>0$ describes the strength of this noise.

Finally, according to Fourier's law, the heat transfer equation in this study is expressed by: 


$$
\frac{\partial T_{l}^{d}}{\partial t}=\kappa_{l} \nabla^{2} T_{l}^{d}
$$

where $\kappa_{l}$ is the thermal diffusivity of the liquid phase.

\subsubsection{Boundary conditions}

The boundary conditions for solving Eqs. (5) and (6) are the following. A zero-mass flux and an adiabatic boundary condition are applied at the bottom substrate. Moreover, we consider that the substrate is neutral with respect to the two components of the binary mixture $[41,68]$, in that there is no wetting preference for either of them. These 3 boundary conditions are respectively written as:
n. $\nabla \mu=0$
n. $\nabla T_{l}^{d}=0$
n. $\nabla \emptyset_{\mathrm{sv}}=0$

where $\mathbf{n}$ is the unit vector normal to the substrate.

Considering that the inert gas absorption in the liquid is negligible and that only the solvent is volatile, the evaporation flux $J$ calculated at the liquid-gas interface takes the following form [69-71]:

$$
\text { n. } M \nabla \mu=J\left(1-\emptyset_{\mathrm{sv}}\right)
$$

where $\mathbf{n}$ is the unit vector normal to the gas-liquid interface.

Concerning the physics of the evaporation at the interface, we consider a simple representation in which the evaporation flux is directly proportional to the volume fraction of the volatile component at the liquid-gas interface [72-75]:

$$
J=\left.J_{o} \emptyset_{\mathrm{sv}}\right|_{z=h_{l}^{d}}
$$

where $J_{o}$ is a global mass transfer coefficient and $h_{l}^{d}(t)$ is the thickness of the liquid layer.

Assuming that the liquid-gas interface remains flat during the evaporation, the time evolution of $h_{l}^{d}(t)$ due to the evaporation is described by:

$$
\begin{aligned}
& \frac{d h_{l}^{d}}{\partial t}=-\bar{J} \\
& \bar{J}=\left.J_{o} \bar{\emptyset}_{\mathrm{sv}}\right|_{z=h_{l}^{d}}
\end{aligned}
$$


where $\bar{\emptyset}_{\mathrm{sv}}$ and $\bar{J}$ are the average of the solvent volume fraction and evaporation flux at the liquid-gas interface.

Moreover, we consider that the liquid-gas interface is neutral with respect to the two components of the binary mixture and one thus gets a condition similar to Eq. (9):

$$
\text { n. } \nabla \emptyset_{\mathrm{sv}}=0
$$

Assuming the characteristic thermal time in the gas is much smaller than in the liquid, we can consider that the temperature of the upper gas phase is homogeneous, constant and equal to the ambient temperature. Therefore, the energy conservation at the liquid-gas interface takes the following form [70, 71]:

$$
J L \rho_{l}=-\alpha_{l} \frac{\partial T_{l}^{d}}{\partial z}
$$

where $\rho_{l}$ and $\alpha_{l}$ are respectively the density and thermal conductivity of the liquid and $L$ is the latent heat of evaporation of the solvent (all these parameters are assumed constant).

Note that we also introduce periodic boundary conditions in the horizontal $x$ direction, in a domain of width equal to $w$ (see Fig. 2).

\subsubsection{Initial conditions}

The initial concentration and temperature fields in the film are assumed spatially uniform and chosen in such a way that the system is in the stable region (i.e. the representative point is above the binodal curve).

\subsection{Non-dimensional equations}

It is convenient to rewrite the equations in a non-dimensional form. The following scaling factors are used for this purpose. The length scale is taken to be $\varepsilon$ and $\varepsilon^{2} / M$ is used as the time scale. The local dimensionless temperatures $T_{l}$ of the liquid is defined as $T_{l}=\left(\frac{T_{l}^{d}-T_{c r}^{d}}{T_{c r}^{d}-T_{c}^{d}}\right)$. In these new units, the equations take the form

$$
\begin{aligned}
& \frac{\partial \emptyset_{\mathrm{sv}}}{\partial t}=\nabla^{2}\left(-\nabla^{2} \emptyset_{\mathrm{sv}}+2\left(-1+2 \emptyset_{\mathrm{sv}}\right)\left(\left(1-2 \emptyset_{\mathrm{sv}}\right)^{2}+T_{l}\right)\right)+\sigma \eta_{\mathrm{s}} \\
& \frac{\partial T_{l}}{\partial t}=L e \nabla^{2} T_{l}
\end{aligned}
$$


where $L e=\frac{\kappa_{l}}{M}$ is a Lewis number and $\eta_{\mathrm{s}}$ is the dimensionless form of $\xi_{\mathrm{s}}$, with $\left\langle\eta_{\mathrm{s}}(r, t)\right\rangle=0$ and $\left\langle\eta_{\mathrm{s}}(r, t) \eta_{\mathrm{s}}\left(r^{\prime}, t^{\prime}\right)\right\rangle=-\nabla^{2} \delta\left(r-r^{\prime}\right) \delta\left(t-t^{\prime}\right)$. The boundary conditions at the bottom are:

$$
\begin{aligned}
& \text { n. } \nabla\left(-\nabla^{2} \emptyset_{\mathrm{sv}}+2\left(-1+2 \emptyset_{\mathrm{sv}}\right)\left(\left(1-2 \emptyset_{\mathrm{sv}}\right)^{2}+T_{l}\right)\right)=0 \\
& \text { n. } \nabla T_{l}=0 \\
& \text { n. } \nabla \emptyset_{\mathrm{sv}}=0
\end{aligned}
$$

At the top, the boundary conditions become:

$$
\begin{aligned}
& \text { n. } \nabla\left(-\nabla^{2} \emptyset_{\mathrm{sv}}+2\left(-1+2 \emptyset_{\mathrm{sv}}\right)\left(\left(1-2 \emptyset_{\mathrm{sv}}\right)^{2}+T_{l}\right)\right)=P e \emptyset_{\mathrm{sv}}\left(1-\emptyset_{\mathrm{sv}}\right) \\
& \frac{d h_{l}}{d t}=-P e \bar{\emptyset}_{\mathrm{sv}} \\
& \text { n. } \nabla \emptyset_{\mathrm{sv}}=0 \\
& \beta P e \emptyset_{\mathrm{sv}}=-\frac{\partial T_{l}}{\partial z}, \beta=\frac{M L \rho_{l}}{\alpha_{l}\left(T_{c r}^{d}-T_{c}^{d}\right)}
\end{aligned}
$$

where $h_{l}(t)$ is the dimensionless height of the liquid layer, whose initial value is

written $H_{l} . \beta$ is the dimensionless latent heat of evaporation and $P e=J_{o} /\left(\frac{M}{\varepsilon}\right)$ is a Péclet number. This non-dimensional number allows comparing the evaporation with the mobility coefficient, which describes both the diffusion when the components are miscible and their separation in the miscibility gap. Note that the dimensionless domain width is written $W$.

\subsection{Numerical implementation}

The numerical simulations have been carried out using the commercial CFD software COMSOL Multiphysics. This software allows solving any partial differential equations (PDE) system using the finite-element method (FEM) and adaptive time steps [76]. FEM has been applied widely for solving the Cahn-Hilliard equation [77-79], notably via COMSOL Multiphysics [68, 80-83], and for investigating solvent evaporation induced phase separation in ternary systems [74]. For time stepping, a Backward Differential Formula (BDF) method was implemented with time steps taken by the solver [76]. In this regard, the solver automatically modifies the time step to satisfy the convergence criteria.

From a computational point of view, an evaporation process is a problem with a moving boundary, since the liquid thickness decreases over time $\left(h_{l}=h_{l}(t)\right)$. COMSOL Multiphysics is able to deal with moving boundaries [84-86] and the Arbitrary Lagrangian-Eulerian (ALE) module implemented in COMSOL Multiphysics was used to manage the moving interface [76]. The phase field model and the temperature equation have been coupled with moving mesh, allowing an automatic remeshing procedure during the time evolution of the physical domain. A mesh-independency test has been carried out to determine the optimal number of 
elements and to ensure that the solution is independent of the mesh size. We generated unstructured meshes using COMSOL Multiphysics (free triangular meshes) for different non-dimensional widths of the elements: 1/3, 1/4, 1/5, 1/6 and 1/8. These choices correspond to about $3,4,5,6$ and 8 elements across the capillary width. In Fig. 3, the time evolution of the average value of the solvent volume fraction at the liquid-gas interface, for $\mathrm{Pe}=0.02, L e=20, \beta=0.4, \sigma=0.005$, a dimensionless width $W=80$ and an initial dimensionless height $H_{l}=20$, is evaluated for these different meshes. The initial volume fraction of the solvent and the initial temperature of the liquid phase are $\emptyset_{\mathrm{sv}}=0.9$ and $T_{l}=0.2$, respectively. Our analysis clarifies that decreasing the dimensionless size of the elements from $1 / 4$ to $1 / 8$ hardly changes the results. Therefore, to minimize the computational time, we carried out the rest of our simulations with a dimensionless size of the elements equal to $1 / 5$.

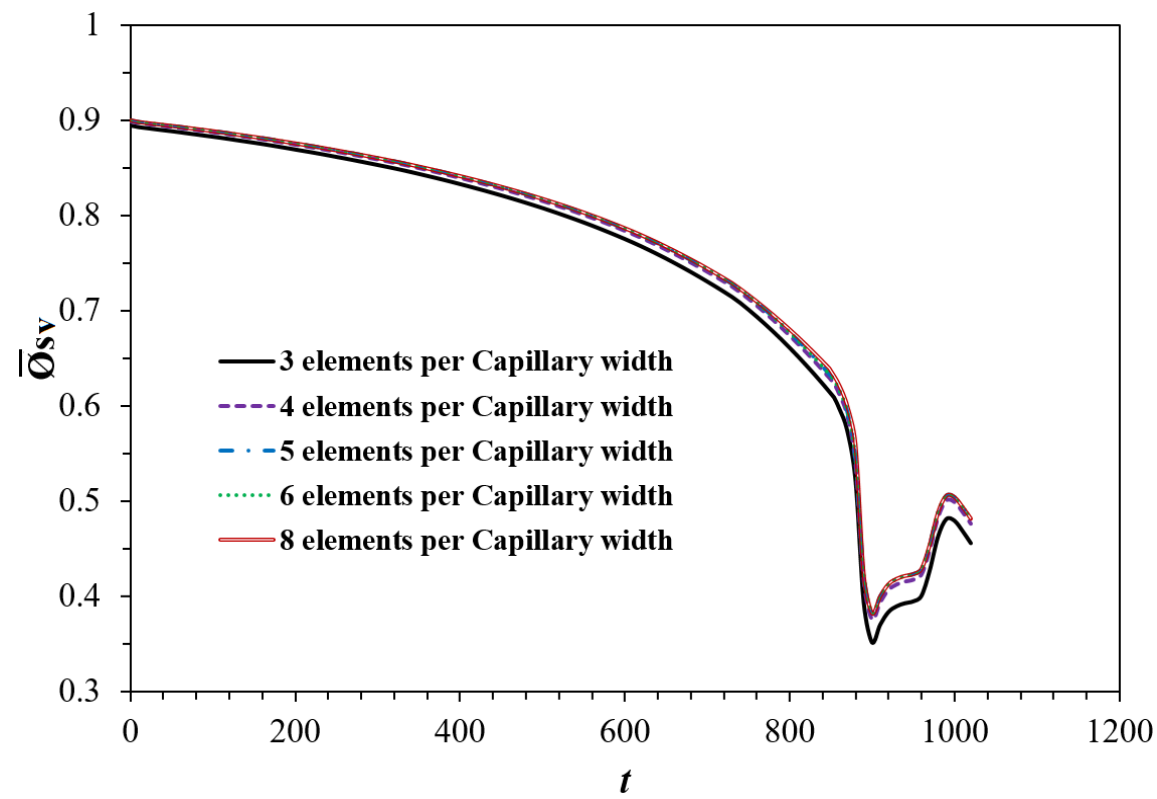

Fig. 3. Time evolution of the average value of the solvent volume fraction at the liquid-gas interface, for different mesh widths.

\subsection{Experimental set-up}

To experimentally investigate the mechanism of 2-D phase separation in a thin evaporating film of a partially miscible binary mixture, a Hele Shaw cell was used with a double telecentric setup, as shown in Fig. 4. The Hele Shaw cell confines a liquid in a shallow volume with an open upper side. In this research, the Hele Shaw cell was built using two Plexiglas plates, and the confinement at the bottom and along the lateral sides was realized thanks to a thin elastic strip (see Fig. 5). To ensure a uniform gap of $1.4 \mathrm{~mm}$ between the plates, the Plexiglas plates and the elastic band 
were maintained using an aluminum casing and $16 \mathrm{M} 3$ bolts. A LED light (1) passing through a telecentric lens (2) goes through the Hele Shaw Cell (4) placed on the sample holder (3) as shown in Fig. 4. Before being captured by the camera (6), the light beam goes through another adjustable telecentric lens (5). The two lenses with adjustable openings make the visualization technique very sensitive to the change of refractive index, which is relevant here as the partially miscible liquids refract light differently.

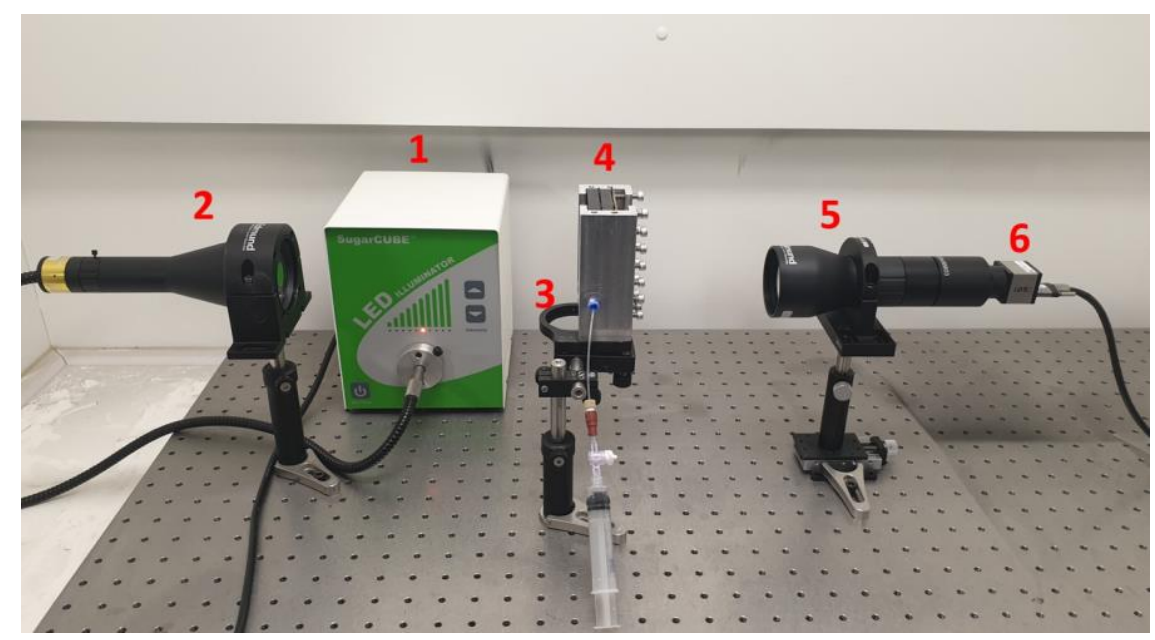

Fig. 4. Double telecentric setup; 1) LED light source, 2) adjustable telecentric lens connected to light source, 3) sample holder, 4) Hele Shaw cell, 5) adjustable telecentric lens connected to camera and 6) camera.
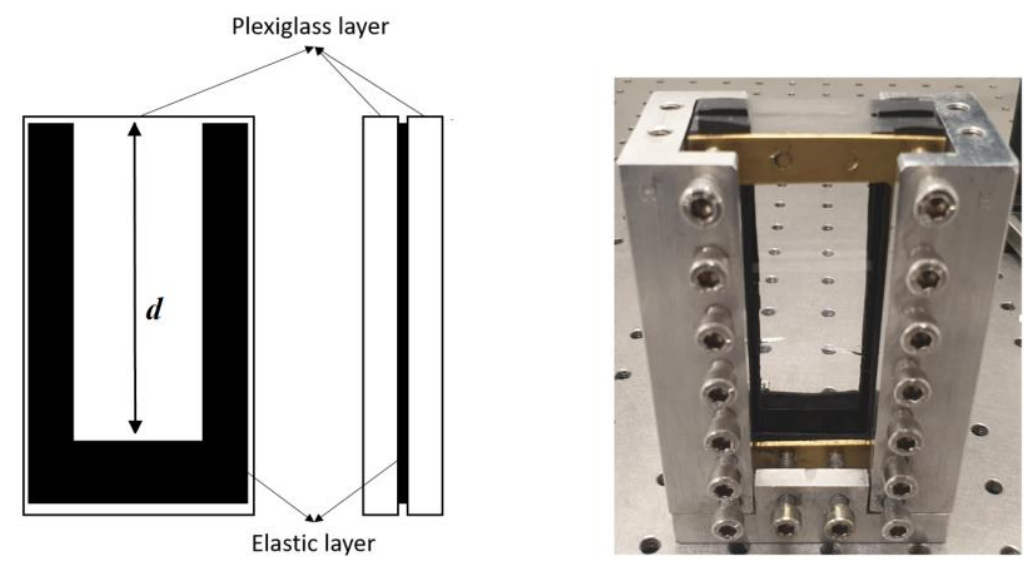

Fig. 5. Hele Shaw cell.

The liquids used in this work are n-hexane and 2-(2-Ethoxyethoxy)ethanol, also known as Diethylene Glycol Monoethyl Ether (DGME), used as purchased from Sigma-Aldrich company. The components are partially miscible and there is a considerable difference between their volatility [43], which allows considering that 
only the n-hexane evaporates. The binodal curve defining the miscibility gap in the phase diagram of the two partially miscible liquids is available in the literature [87]. Before each test, the Hele Shaw cell was disassembled, washed by ethanol and optical tissue and left to dry. The mixture was injected from the bottom of the cell using a syringe connected to a Polytetrafluoroethylene tube fixed to the cell. The tests were performed during different days at atmospheric pressure, for an initial liquid thickness of $10 \mathrm{~mm}$, and with the room temperature between $19^{\circ} \mathrm{C}$ and $21^{\circ} \mathrm{C}$. This is above the upper critical temperature of the system $\left(6.3^{\circ} \mathrm{C}\right.$ [87]). The relative humidity of the air was between $43 \%$ and $52 \%$. Each test was repeated at least three times. The distance from the bottom of the Hele Shaw cell to the open air is denoted $d$ and this distance has been varied from one test to another in order to change the evaporation rate (see below).

\section{Results and discussion}

The fluids properties used in the model are those corresponding to our experiments and already described previously in [43] (see section 3 in [43] for more details). They give $L e \approx 20$ and $\beta \approx 0.4$ and these two values are used throughout this work. Note that we assume that the values of these properties are not substantially modified by the concentration and temperature changes in the mixture induced by the evaporation and thus that they can be considered as constant during the time evolution of the system. We consider a noise strength $\sigma=0.005$, to make sure that phase separation occurs in the system [88]. Note that we also examined a stronger noise $(\sigma=0.05)$ for our simulations and it showed that increasing noise does not change significantly the results and the morphology of phase separation.

\subsection{Interplay between the evaporation rate and the phase separation}

Evaporation is the key phenomenon driving the phase separation in the studied system. Indeed, since the system is initially in the stable region, evaporation leads the system to approach the binodal curve by decreasing the temperature and increasing the solute concentration close to the upper surface (see Fig. 1). Then, the binodal curve, and later the spinodal one, are crossed, which makes the system unstable and allows the beginning of phase separation. Note that some initial conditions (for instance high temperature, see [43] for more details) could prevent the system from crossing these curves. In such situations, phase separation does not occur. Note also that, depending on the initial temperature and concentration, the system will reach the demixing region at different locations in the phase diagram (i.e. at different temperature and concentration), and phase separation will thus start at a temperature that varies with the initial conditions. This point is very important, because it is well known that phase separation proceeds faster at lower temperatures, 
due to the higher driving force for phase separation (see [89-91] for more details on the effect of different quench depths in the phase separation process).

For the simulations presented in this section, the computational domain is rectangular, with a dimensionless width $W=80$ and an initial dimensionless height $H_{l}=20$. Figs. 6 and 7 describe the interplay between evaporation and the phase separation dynamics for an initial solvent volume fraction $\emptyset_{\mathrm{sv}}=0.9$ and an initial temperature $T_{l}=0.2$. Different evaporation rates are considered by choosing the following different values of the Péclet number: $\mathrm{Pe}=0.002, \mathrm{Pe}=0.02$, and $\mathrm{Pe}=$ 0.08.)

For $\mathrm{Pe}=0.002$ (Fig. 6), which corresponds to a low evaporation rate, the system remains almost vertically uniform and thus the phase separation occurs everywhere across the bulk of the thin film when the system enters the miscibility gap.

For $\mathrm{Pe}=0.02$, which corresponds to a moderate evaporation rate, the liquid close to the liquid-gas interface enters the spinodal before the bottom of the layer (see the time labels in Fig. 7-c). For this reason, the spinodal instability takes place only in the upper part of the system, which creates a lateral structure in the film, characterized by a well-defined wavelength. Then as time increases, this lateral structure progressively invades the whole thickness of the fluid. In Fig. 7-c, the paths in the phase diagram describing the time evolution of the average values of the temperature and of the solute volume fraction at the liquid-gas interface and at the substrate are presented. We clearly see in this figure that the top of the layer enters the unstable region before the bottom of the layer. Moreover, we see that the evolution of the average solute volume fraction at the liquid-gas interface and at the bottom of the film is not monotonous. Notably, while the bottom of the film is in the metastable region, we observe a decrease of the solute concentration there (i.e. an increase of the solvent concentration). It is due to the phase separation taking place at the top of the layer, transferring some solvent towards the bottom of the film. Finally, in the case of a "fast" evaporation with $\mathrm{Pe}=0.08$, phase separation appears only in the vertical direction as a result of the concentration gradient imposed by the evaporation. The spinodal instability and the resulting formation pattern characterized by a typical wavelength do not occur at liquid-gas interface and the so-called lamellar morphology is observed, with a thin horizontal skin of solute in the upper part of the layer. As evaporation continues, the lower part of the layer enters the unstable region (see Fig. 7-d) and a spinodal instability takes place in the neighborhood of the substrate, similar to the spinodal instability at the liquid-gas interface for the lateral structure. Some solvent-rich droplets are formed but they finally disappear. 


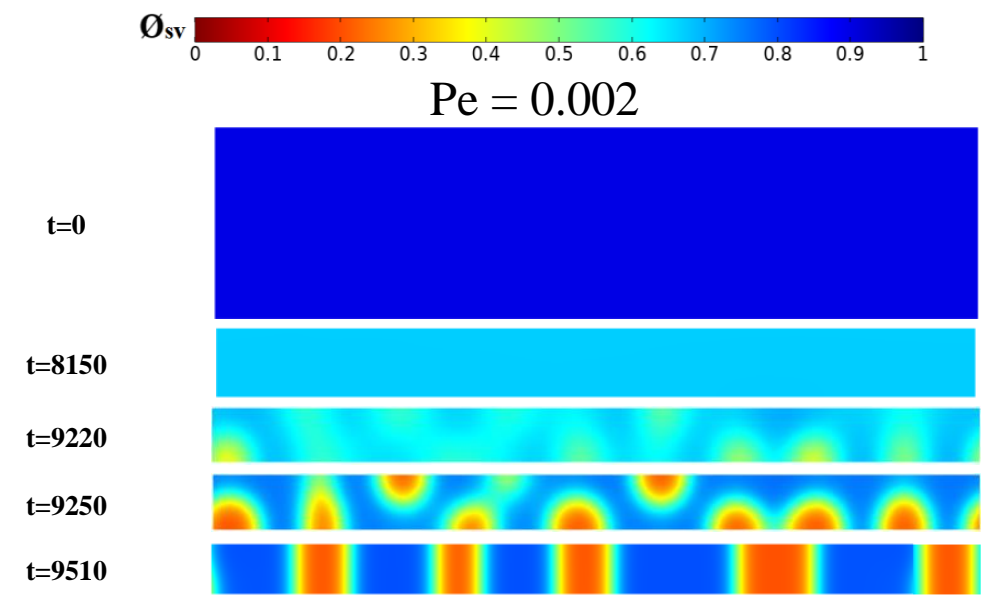

Fig. 6. Time evolution of the phase separation morphology for $\mathrm{Pe}=0.002 . H_{l}=20, W=80, \emptyset_{\mathrm{sv}}=0.9$ and $T_{l}=0.2$ at $t$

$$
=0 \text {. }
$$

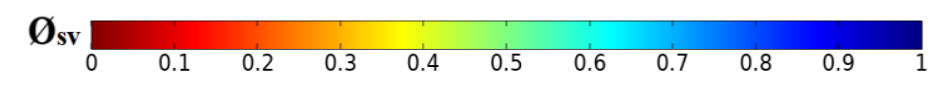

$\mathbf{a}$

$\mathbf{t}=\mathbf{0}$

$t=810$

$\mathrm{t}=\mathbf{8 9 0}$

$t=895$

$\mathbf{t}=\mathbf{9 1 0}$

$\mathbf{t}=\mathbf{9 4 0}$

$\mathrm{t}=\mathbf{9 7 0}$

$\mathrm{t}=\mathbf{1 0 2 0}$
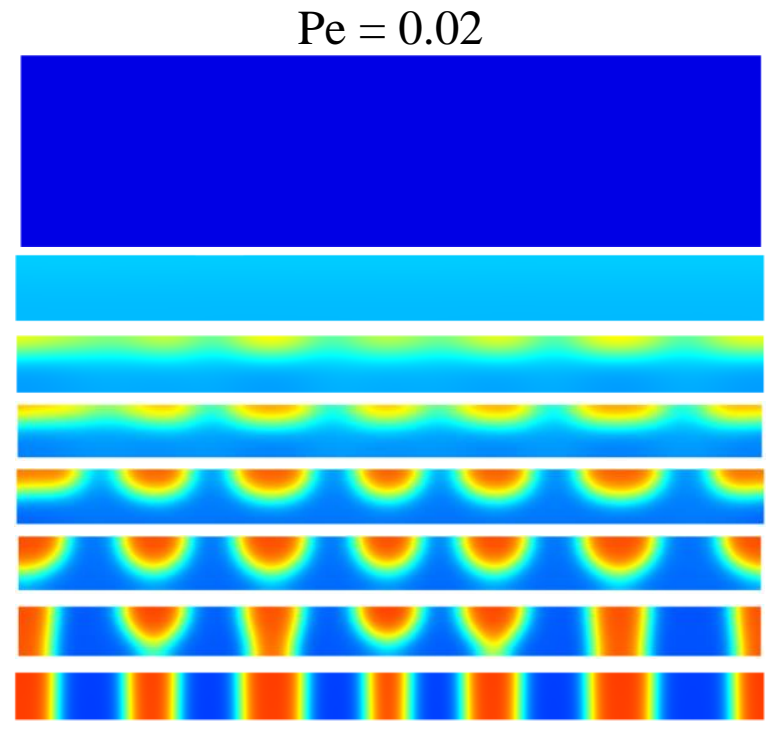

c

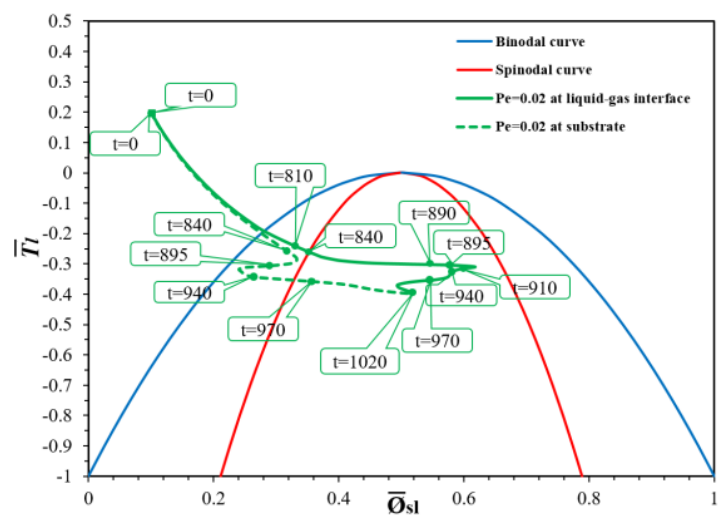

b

$\mathrm{Pe}=0.08$

$\mathbf{t}=\mathbf{0}$

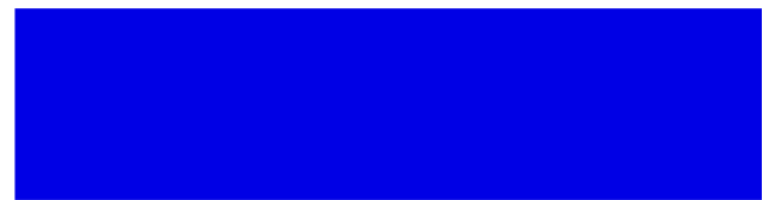

$t=200$

$\mathrm{t}=\mathbf{2 3 0}$

$t=260$

$\mathbf{t}=\mathbf{3 1 0}$

$t=326$

$t=342$

$t=386$

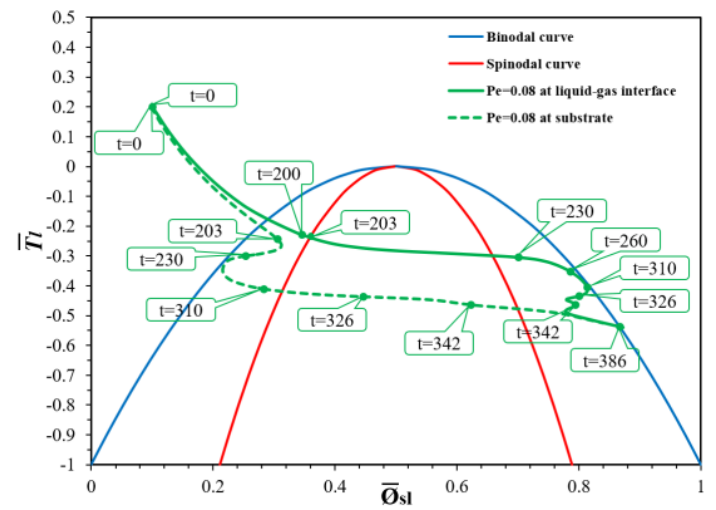


Fig. 7. a and b: time evolution of the phase separation morphology. $\mathrm{c}$ and d: paths in the phase diagram describing the evolution with time of the average values of the temperature and of the solute volume faction at the liquid-gas interface and at the substrate. $\mathrm{Pe}=0.02$ and $0.08, \emptyset_{\mathrm{sv}}=0.9$ and $T_{l}=0.2$ at $t=0, H_{l}=20, W=80$.

Another interesting global indicator of the evolution of the system is the evaporation flux Pe $\bar{\emptyset}_{\text {sv }}$. This quantity, rescaled by Pe, is plotted versus time in Fig. 8 for three values of the Péclet number: $\mathrm{Pe}=0.02,0.03$, and 0.08 . As expected, the evaporation flux decreases over time due to the removal of solvent at the liquid-gas interface. However, the decrease of the evaporation flux is not monotonous and some sudden changes are also observed, which are emphasized by circles in the figure. These changes are further discussed in the next section.

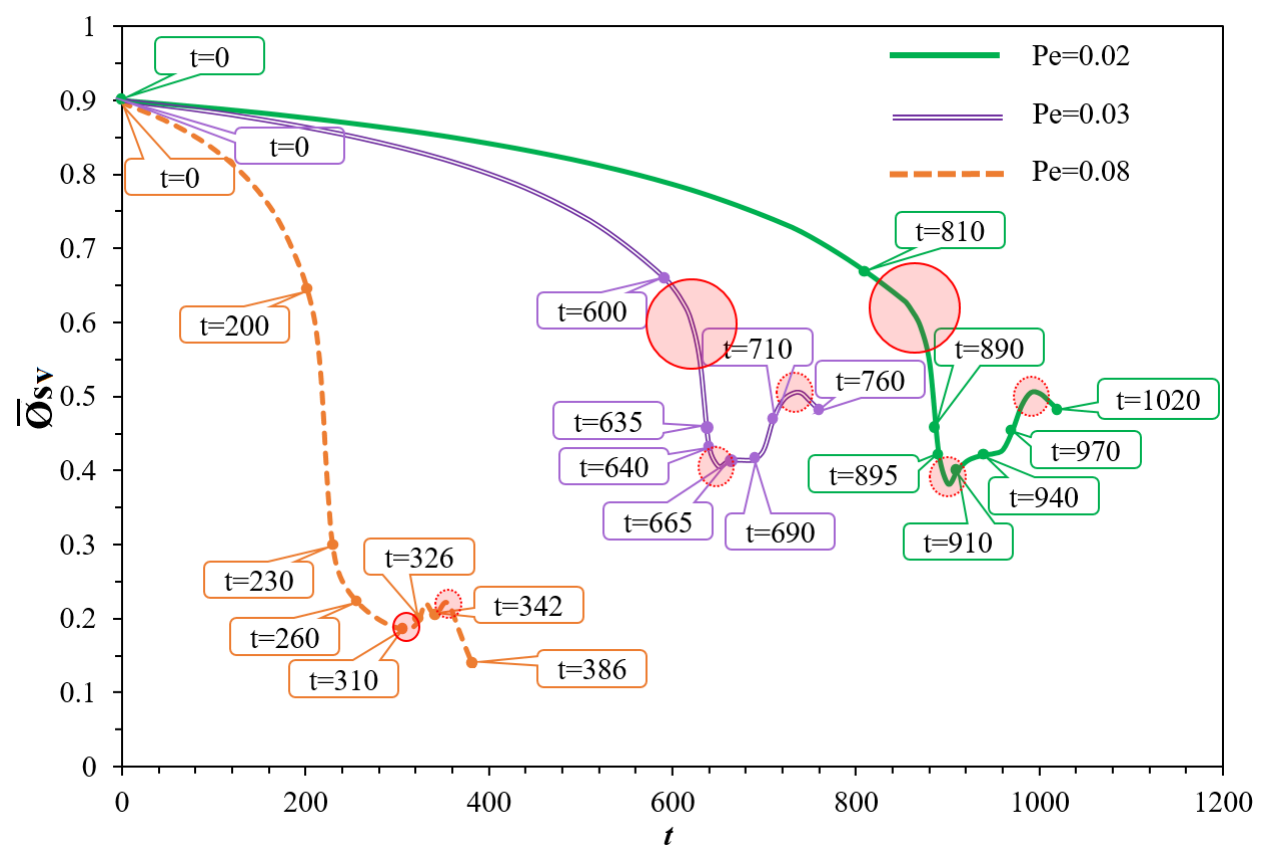

Fig. 8. Time evolution of the average evaporation flux at the gas-liquid interface, rescaled by Pe, for three values of the Péclet number: $\mathrm{Pe}=0.02,0.03$, and $0.08 . \emptyset_{\mathrm{sv}}=0.9$ and $T_{l}=0.2$ at $t=0, H_{l}=20, W=80$.

\subsection{Beginning of the phase separation}

As already mentioned, the Péclet number is a measure of the competition between evaporation and phase separation. The results shown above for 3 values of Pe clearly emphasize the important role of this parameter on the behavior of the system.

Low evaporation rates have been considered first, with $\mathrm{Pe}=0.002$. In that situation, the system is almost uniform in the vertical direction when it enters the unstable region in the phase diagram. For this reason, the spinodal instability takes place through the whole layer at the same time, as observed in Fig. 6. Note that this expected behavior has already been studied in [53], where the isothermal demixing of a polymer-solvent mixture, induced by the solvent evaporation, was analyzed at 
a very low evaporation rate. This slow evaporation and the corresponding phase separation are not analyzed further in the present paper.

The case of a moderate evaporation rate, with $\mathrm{Pe}=0.02$, is described by Fig. 7-a. In this situation, a moderate vertical concentration gradient is present in the liquid film. Its upper part enters the unstable region while its bottom part is in the metastable region. For this reason, the spinodal instability takes place only close to the top surface, which gives rise to the lateral morphology that appears in Fig. 7-a for $t$ 890. The appearance of this instability is also related to a rather sudden decrease of the evaporation rate versus time, which is emphasized by Fig. 8 for Pe $=0.02$ and 0.03 (see the first red circle for $\mathrm{Pe}=0.02$ and 0.03 ). As already mentioned above, the instability is also related to an increase of the solvent concentration close to the bottom substrate.

The Péclet number has also an interesting effect on the wavelength (or wavenumber) that characterizes the lateral pattern generated by the spinodal instability in the upper part of the system. In our numerical approach, the wavelength $\lambda$ can be estimated from the number $n$ of cells that appear close to the interface, with $\lambda=W / n$. To have a more precise numerical estimation of this quantity, it is of course preferable to decrease the constraining influence of the (periodic) lateral boundaries of the layer and we use $W=160$ for the results presented in the present discussion. Using the same initial conditions as previously $\left(H_{l}=20, \emptyset_{\mathrm{sv}}=0.9\right.$ and $\left.T_{l}=0.2\right)$, we consider three values of the Péclet number, $\mathrm{Pe}=0.02,0.03$, and 0.04 , and the associated patterns observed right after the instability are represented in Fig. 9. The numbers of cells are respectively 12,14 and 15, which emphasizes that the increase of the Péclet number (i.e. of the evaporation rate) corresponds to an increase of the observed wavenumber.

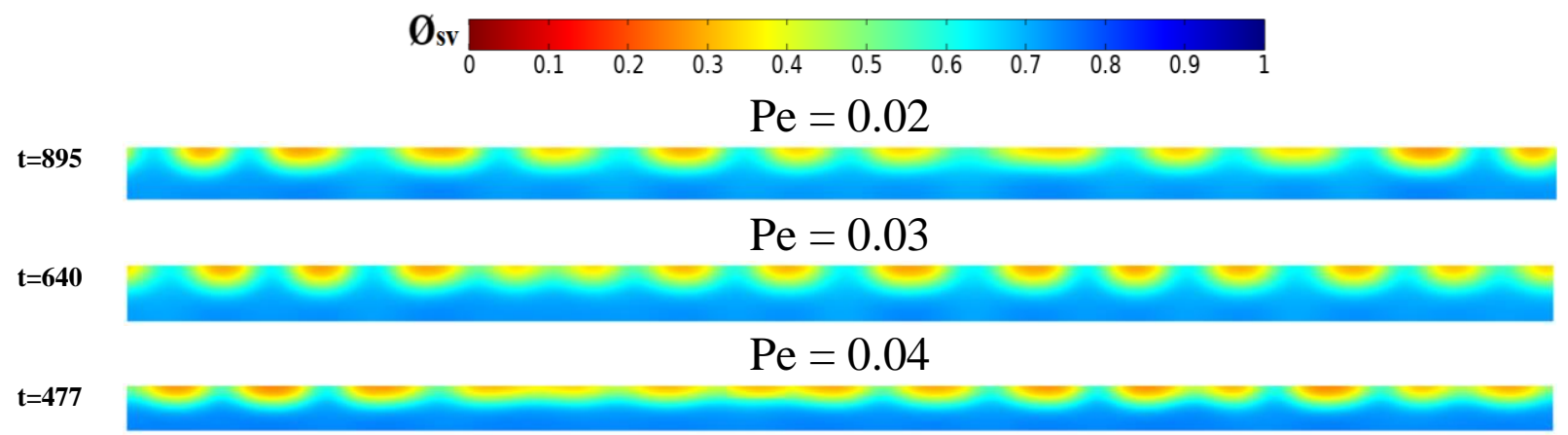

Fig. 9. Phase separation pattern for three different values of the Péclet number: $\mathrm{Pe}=$ $0.02,0.03$, and $0.04 . \emptyset_{\mathrm{sv}}=0.9$ and $T_{l}=0.2$ at $t=0, H_{l}=20, W=160$.

Finally, a Péclet number equal to 0.08 corresponds to a large evaporation rate. In that situation, Fig. 7-b shows that the phase separation occurs only in the vertical direction, as a consequence of the vertical gradient imposed by the evaporation. No spinodal instability appears in the upper part of the system when it enters the unstable region and a so-called lamellar morphology is created. Note that this layered 
morphology was also observed in [52] for the isothermal phase separation induced by a fast solvent evaporation of a single polymer-solvent binary mixture in a cylindrical geometry. This behavior can be explained as follows. First, it is interesting to note generally that a spinodal instability close to the upper surface induces the development of horizontal concentration gradients. Since the evaporation flux is proportional to the solvent concentration at the interface, the evaporation then reduces the horizontal concentration gradients between the solventrich and solute-rich areas and thus participates in the damping of the perturbations. This argument clearly emphasizes the competition between separation and evaporation and the important role of the Péclet number. It is also interesting to note the rather complex role played by evaporation in the phenomena under analysis. On the one side, evaporation brings the fluid in the demixing area, which allows for the spinodal instability, and it is worth noting that the path in the phase diagram depends only slightly on the Péclet number, i.e. on the intensity of the evaporation. On the other side, evaporation can damp the perturbations generated by the instability and, if $\mathrm{Pe}$ is large enough, this damping can finally prevent the appearance of the lateral morphology, which allows understanding the lamellar morphology for Pe $=0.08$. Interesting and important interpretations of the above results can be obtained by considering a qualitative linear stability analysis, with an approach which is in fact rather close to that used in [92]. As a first step, let us briefly recall the linear CahnHilliard theory of spinodal instability for quenching experiments in partially miscible binary mixtures $[13,93-95]$. When such a mixture is suddenly quenched into the miscibility gap, a band $\left[0, k_{c}\right]$ of wavenumbers becomes unstable, with:

$$
k_{c}^{2}=-\left(\frac{\partial^{2} f_{0}}{\partial \emptyset_{s v}{ }^{2}}\right)
$$

Remember also that the dominant wavenumber $k_{m}$, and its growth rate $\omega_{m}$, which is the maximum value over the unstable band, are given by:

$$
k_{m}^{2}=\frac{1}{2} k_{c}^{2} \quad \omega_{m}=\frac{1}{4} k_{c}^{4}
$$

The situation analyzed in our work is of course more complex than quenching, notably because the entrance in the miscibility gap is progressive. Therefore, the analysis to carry out should examine the stability of a time-dependent (and zdependent) reference profile and determine, at each instant, the growth rates of $z$ dependent perturbations. Such approaches can be based on the so-called frozen-time assumption and have been developed in other contexts for example in [70, 96, 97]. Instead of this complete analysis that requires rather complex numerical calculations, we develop here a simplified and qualitative approach, that 
nevertheless allows one to obtain interesting trends and general features of the instability mechanism. The argument is based on the fact, during the transient that brings the system in the phase diagram close to the spinodal curve, the representative point of the upper surface of the film is always ahead and closer to the bell curve. Therefore, the upper surface always enters first the instability region and the first growing perturbations always appear at the surface (note that, for the ternary mixtures analyzed in [92], the first unstable zones are not always at the surface). From that moment, the instability begins to develop, while at the same time the representative point of the surface gets deeper inside the spinodal curve and regions below the surface progressively reach the instability region as well. To have an idea of the modes that will be excited during this complex evolution, we will consider the upper surface, with its mean temperature and concentration, as a global indicator of the state of the system and the time evolution of the corresponding point in the phase diagram is used to determine the time dependence of the band of unstable modes of the previously recalled Cahn-Hilliard theory. When the system crosses the spinodal curve on the left (see Fig. 7-c), the band has first a zero width. Then, as time passes, the width of the unstable band increases, and the corresponding $k_{m}$ and $\omega_{m}$ also increase, until the system reaches the maximum $k_{c}^{2}$, i.e. the minimum $\partial^{2} f_{0} / \partial \emptyset_{s v}{ }^{2}$, which corresponds to reaching the critical concentration $\emptyset_{\mathrm{sv}}=0.5$. Besides this point, $k_{c}, k_{m}$ and $\omega_{m}$ start to decrease. For $\mathrm{Pe}=0.02,0.03,0.04$, and 0.08, Fig. 10 shows the time evolution of $k_{m}$ and $\omega_{m}$ for the global indicator introduced above and calculated using Eqs. (24) and (25). It is interesting to stress that the three curves corresponding to $\mathrm{Pe}=0.02,0.03$, and 0.04 are quite similar, except that the time interval $\Delta t$ between the crossing of the spinodal and reaching the maximum of $k_{c}$, $k_{m}$ and $\omega_{m}$ decreases when the evaporation is stronger. This means that when the evaporation rate is larger, the representative point of the surface crosses the miscibility gap at a faster speed and that larger $k_{m}$ are excited sooner, as emphasized by Fig. 10. Note also that the three time intervals $\Delta t$ corresponding to $\mathrm{Pe}=0.02$, 0.03 , and 0.04 are larger than the typical growing time $\tau$ of the perturbations, which can be estimated by calculating $2 \pi$ divided by the maximum value of $\omega_{m}$ in Fig. 10, which gives $\tau \approx 17$. This means that the perturbations have time enough to grow and it is thus possible to observe the appearance of the larger $k_{m}$ that are excited sooner for larger evaporation rates. This also provides an explanation for the numerical results presented in Fig. 9 (note that another example of this effect is mentioned in Section 3.4). Let us also emphasize that for the moderate Péclet numbers considered here, only a very thin layer close to the upper surface truly participates in the initial spinodal instability, whose thickness is less than the wavelength of the patterns. Therefore, only lateral patterns are created by this instability (in contrast, for the very low evaporation rate considered in Fig. 6, some 
vertical structure also appears). Finally, note that for $\mathrm{Pe}=0.08$, Fig. 10 shows that the typical growing time of the perturbations is $\tau \approx 19$. Since this time is larger than the time interval $\Delta t=16$ between crossing the spinodal curve and reaching the critical concentration, one can deduce that the system does not have enough time to create the pattern and no spinodal instability occurs in the system, which is actually what we observed in Fig. 7.b.
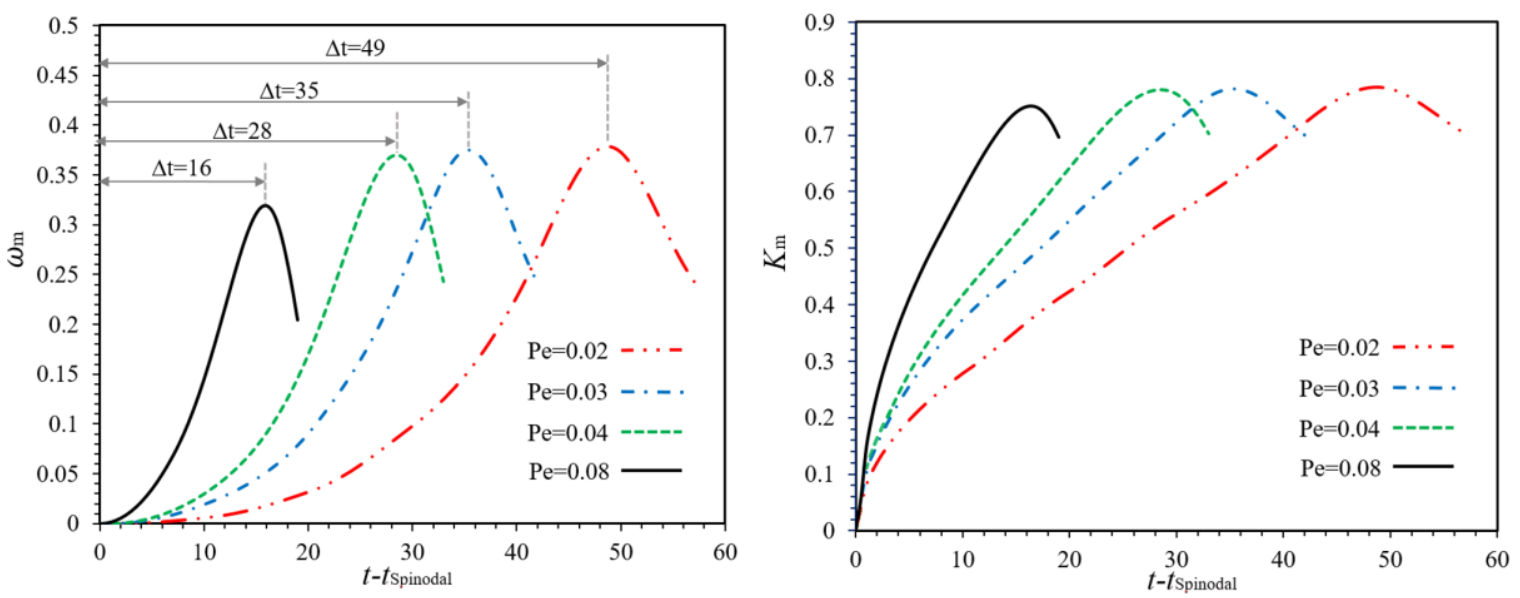

Fig. 10. Growth rate and wavenumber for the average values of the solvent volume fraction and the temperature at the liquid-gas interface ( $t_{\text {spinodal }}$ is the time corresponding to the entry in the unstable miscibility gap) and for $\mathrm{Pe}=0.02,0.03,0.04$, and $0.08 . H_{l}=20, W=160$,

$$
\emptyset_{\mathrm{sv}}=0.9 \text { and } T_{l}=0.2 \text { at } t=0 \text {. }
$$

\subsection{Mid- and long-term evolution of the phase separation morphology}

Here, we analyze the mid- and long-term evolution of the system, (far) after the beginning of the phase separation, described in the previous subsection. We consider only moderate and high evaporation rates, for which phase separation starts close to the liquid-gas interface.

For $\mathrm{Pe}=0.02$ (moderate evaporation rate), we show the time evolution of the morphology and of the evaporation flux in Fig.7-a and in Fig. 8, respectively. As mentioned in subsection 3.2, the first sudden change in the curve describing the evolution with time of the evaporation flux is related to the time when the spinodal instability occurs in the system. This instability, which is emphasized by the first red circle in Fig. 8, close to $t=870$, is associated with a sudden change of the slope of the curve. A sharp decrease of the evaporation flux is then observed, whose duration is of the order of the growing time $\tau$ of the instability. Then a second drastic change of the slope appears in Fig. 8, which is emphasized by the second red circle close to $\mathrm{t}=900$. From that moment, the nonlinear evolution of the phase separation pattern takes places and the solute-rich (red) droplets start to grow (see the growing red zones in Fig. 7-a), while the solvent is rejected towards the bottom or between the red drops (see the blue that gets darker close to the substrate or between the red 
zones). During this stage, the evaporation flux increases but note that this increase is not completely regular because the growth of the solute-rich drops let the red zones touch the substrate one after the other. Finally, close to the third red circle in Fig. 8, all the red drops have reached the bottom and a new evolution stage starts, with a decreasing evaporation flux. However, it is important to stress that this stage of the evolution of the system cannot be studied with our model and we have thus stopped the numerical calculations then. More precisely, the flat surface assumption would artificially squeeze the "red pillars" and prevent a possible dewetting of the substrate and the creation of isolated pure solute droplets on the substrate, as observed experimentally in [43].

For $\mathrm{Pe}=0.08$ (fast evaporation, see Fig.7-b), the evaporation flux decreases importantly when the phase separation starts, owing to the lamellar structure created by the evaporation induced vertical gradient (see Fig. 8). Close to $t=320$, the lower parts of the layer enter the demixing region and a spinodal instability takes place in the neighborhood of the substrate (see also Fig.7-b for $t=326$ ). As a result of this phenomenon, a sudden slope change appears in Fig. 8 (first red circle) and the evaporation flux starts to increase. After some time, the solvent-rich (blue) droplets disappear and the final stage of the evolution starts close to $t=350$ (second red circle), with only solute-rich (red) fluid remaining in the system and the progressive evaporation of the remaining solvent.

Another interesting phenomenon that could appear after the spinodal instability is the coalescence of solute-rich (red) droplets. In fact, the growth of the solute-rich drops takes place both in the vertical and horizontal directions and it is easy to understand that if the thickness of the layer is large enough, the drops could coalesce horizontally before touching the substrate. To illustrate this, we consider the same system as in Fig. 7-a $\left(\mathrm{Pe}=0.02\right.$, initial solvent volume fraction $\emptyset_{\mathrm{sv}}=0.9$ and initial temperature $T_{l}=0.2$ ), but we use three different initial heights $H_{l}=20$ (the height already considered before), $H_{l}=28$, and $H_{l}=38$. The corresponding results are presented in Fig. 11. For the 3 cases, a lateral structure is created first close to the interface. The case $H_{l}=20$ is already discussed above and no coalescence is observed before the red pillars are formed. Fig. 11 then shows that, for $H_{l}=28$, coalescence occurs between some droplets and a smaller number of pillars than for $H_{l}=20$ is formed later. Finally, for $H_{l}=35$ the coalescence of all the drops happens, which gives rise to a secondary lamellar structure. The subsequent evolution of this lamellar structure is then similar to what is observed in Fig. 7-b, with the appearance of a spinodal instability close to the substrate, before only solute-rich (red) fluid remains in the system. Finally note that the possibility of coalescence is linked to an appropriate relation between the dimension of the drops (i.e. the wavelength of the pattern) and the thickness of the layer when the instability takes place. Indeed, Fig. 11-b (that corresponds to an initial thickness equal to 28) shows the coalescence of 
drops when the thickness is around 8, which is of the order of half the wavelength of the pattern (this wavelength is 160/13 13.3, see above).

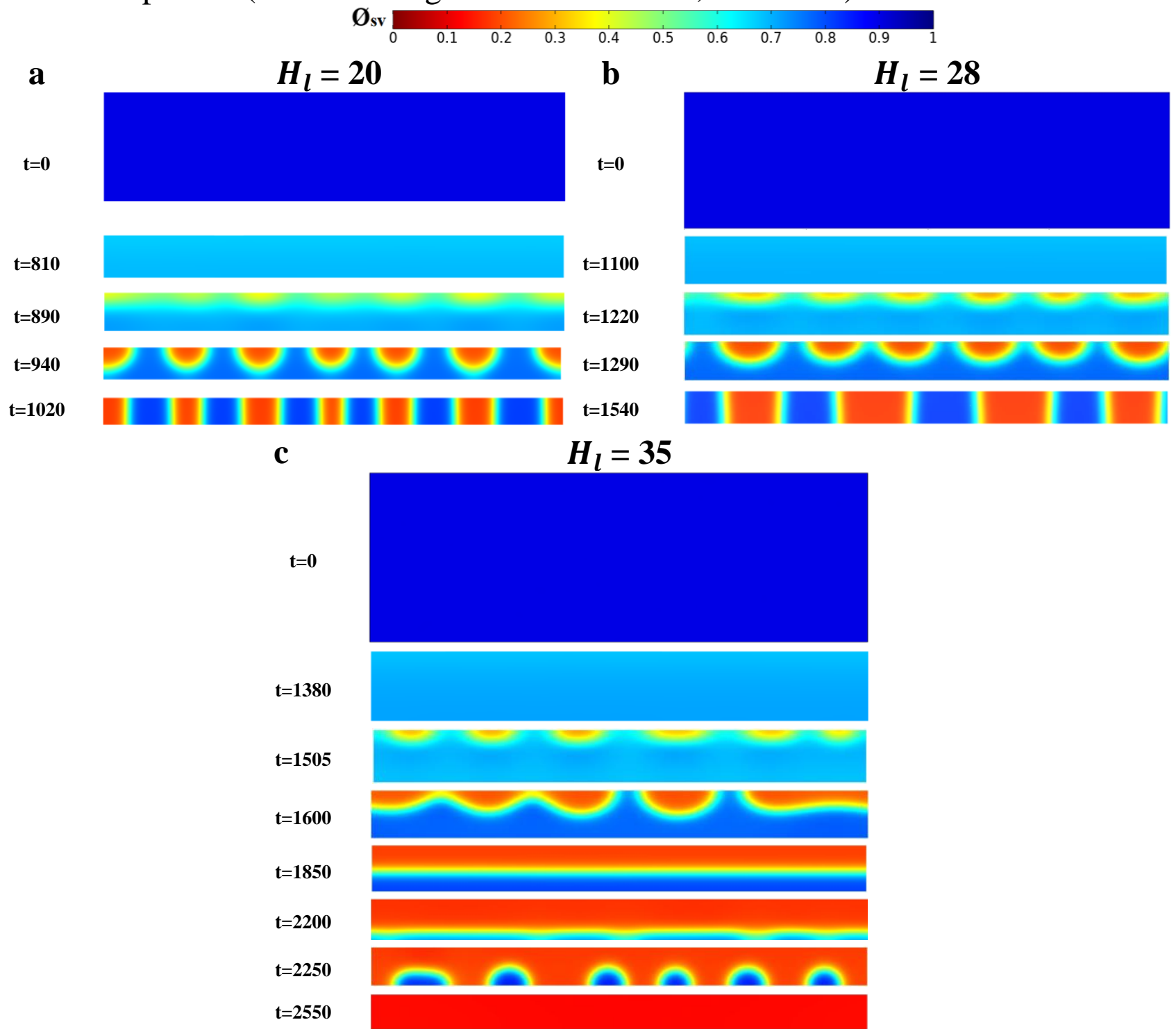

Fig. 11. Time evolution of the phase separation morphology for $\mathrm{Pe}=0.02$ and for three initial dimensionless heights $H_{l}=20,28$, and 35. $\emptyset_{\mathrm{sv}}=0.9$ and $T_{l}=0.2$ at $t=0, W=80$.

\subsection{Influence of the initial conditions on the phase separation morphology}

The initial temperature and concentration are parameters that can influence the phase separation morphology in the binary mixture. In fact, a change of the initial temperature or concentration can lead the system to enter the unstable region at different conditions, which can affect significantly the driving force of the phase separation and its competition with the evaporation. To emphasize this, we consider two examples in this section. First, for $\mathrm{Pe}=0.08$, we consider a change in the initial temperature with respect to the situation in Fig. 7-b. In Fig. 12, we observe that 
reducing the initial temperature to $T_{l}=-0.3$ induces logically an entry in the unstable region at a lower temperature, resulting in higher driving force for the phase separation. Therefore, the phase separation proceeds much faster and eventually overcomes the strong evaporation to form a lateral structure, in contrast to what was observed in Fig. 7-b, for the same Péclet number and for $T_{l}=0.2$. It is also interesting to note that in contrast to what was observed in Fig. 11-a (same initial thickness as in the present situation), coalescence is possible here because the wavelength is smaller than in Fig. 11-a and also because the thickness when the instability takes place is larger (around 9, instead of 6). Considering again the qualitative linear stability analysis for $\mathrm{Pe}=0.08$ and $T_{l}=-0.3$, we have calculated the typical growing time of the perturbations and the time interval to reach the maximum $k_{c}$. The obtained results are $\tau \approx 4$ and $\Delta t=16$, which confirms that the perturbations have time enough to grow and to create the lateral pattern at liquid-gas interface. To check that the mechanism of the competition between phase separation and evaporation is still active here, we have also increased the Péclet number and examined the effect on the morphology. The calculations for $\mathrm{Pe}=0.3, T_{l}=-0.3$ have then revealed a lamellar pattern and no lateral instability was observed, which is completely in line with the values $\tau \approx 6$ and $\Delta t=5$ that we have calculated for this situation. To emphasize again the influence of the Péclet number on the wave length, we have also considered $\mathrm{Pe}=0.04, T_{l}=-0.3$ and 8 droplets have appeared instead of 9 in Fig. 12a, which shows the same trends as in Fig. 9. The above discussion allows also emphasizing the role of temperature in the studied phenomena. The initial temperature determines the entry point of the system in the instability region of the phase diagram and consequently the driving force of phase separation. This in turn modifies the relative importance of evaporation in its competition with phase separation. Temperature can thus be seen as a regulator between the two effects, with the consequence that this easily controlled experimental parameter directly influences the morphology that appears in the film. From our results, it is deduced that decreasing temperature favors in fact the appearance of a lateral structure (we have seen above that $\mathrm{Pe}=0.08$ gives rise to a lamellar structure for $T_{l}=0.2$, while a lateral pattern is obtained for $T_{l}=-0.3$ ). Let us also insist that even if temperature has a clear role in the creation of the morphology, its influence on the spinodal instability itself is minute. Indeed, when this instability takes place, the amplification of the perturbations is exponential and thus very fast and the instability can thus be considered as almost isothermal. This is emphasized by the different paths of the system in the phase diagram, for which the trajectory inside the miscibility gap is fast and almost horizontal. 

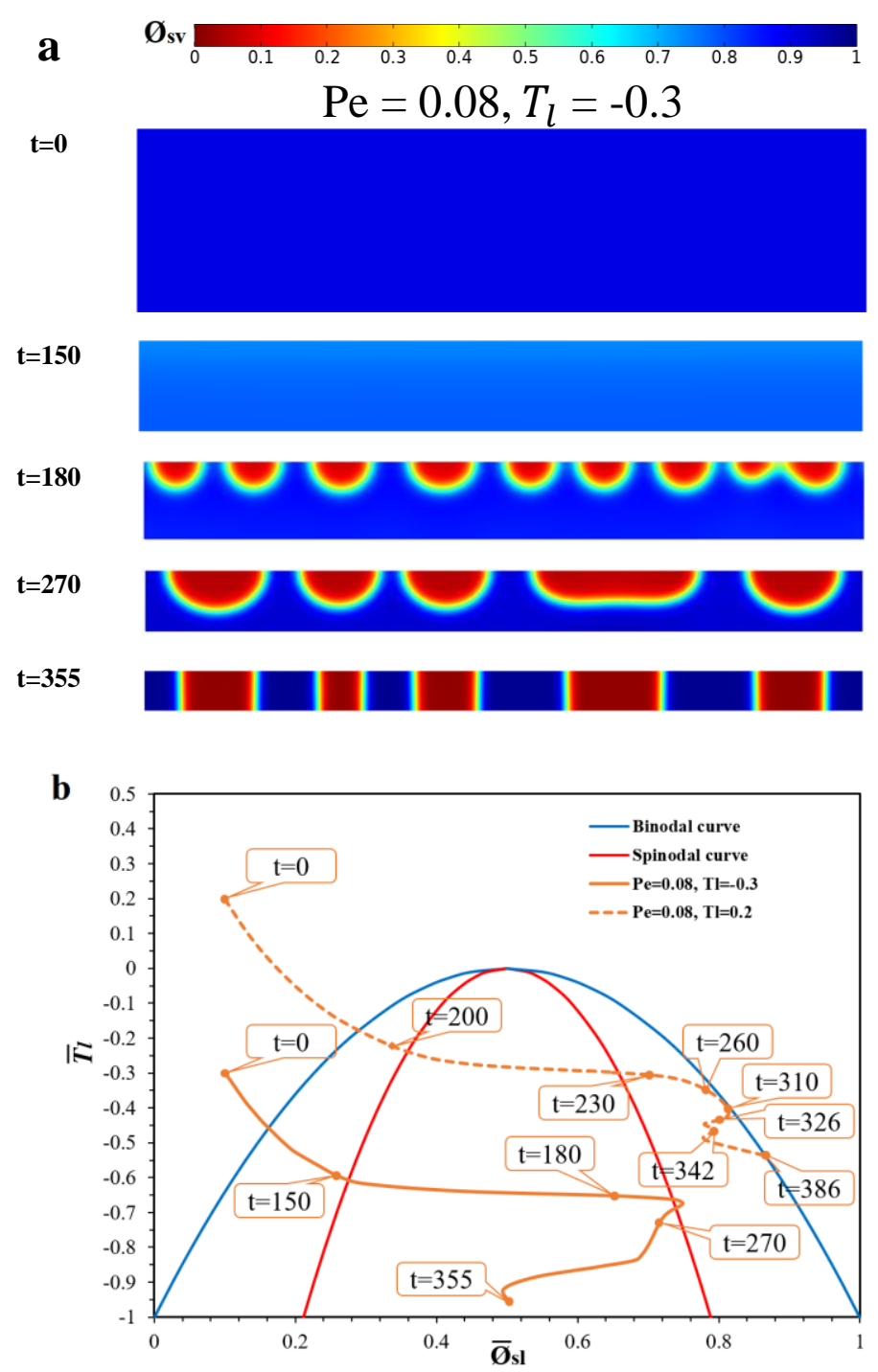

Fig. 12. a: time evolution of the phase separation morphology for $\mathrm{Pe}=0.08$ and $T_{l}=-0.3$. b: path in the phase diagram describing the evolution with time of the average values of the temperature and of the solute volume faction at the liquid-gas interface for $T_{l}=-0.3$ and $T_{l}=$ 0.2. $H_{l}=20, W=80, \emptyset_{\mathrm{sv}}=0.9$ (initial value).

The second example that emphasizes the importance of initial conditions consists in changing the initial concentration with respect to the case of Fig. 7-a $\left(\mathrm{Pe}=0.02, \emptyset_{\mathrm{sv}}\right.$ $=0.9$ ). The corresponding results, for an initial concentration for $\emptyset_{\mathrm{sv}}=0.85$, are represented in Fig. 13. They show that the reduced initial concentration of the solvent leads to a different entry point in the miscibility gap, at a higher temperature, and thus a reduced driving force for the separation in comparison with to the case with $\emptyset_{\mathrm{sv}}=0.9$. Consequently, the phase separation is overcome by the evaporation and a lamellar pattern is obtained (in contrast with Fig. 7-a). Note that we have also calculated the typical growing time of the perturbations and the time interval to reach the maximum $k_{c}$, with $\tau \approx 51$ and $\Delta t=49$, which indicates that not enough time 
is available to build a lateral pattern. The competition between phase separation and evaporation was then again tested, by decreasing the Péclet number in the case $\emptyset_{\mathrm{sv}}=$ 0.85 , and for $\mathrm{Pe}=0.01$, a lateral structure was obtained $(\tau \approx 46$ and $\Delta t=95)$. As a final remark, let us also mention that the above analysis indicates that reducing the initial concentration of the solvent, i.e. increasing the concentration of the nonvolatile solute, favors the lamellar morphology.
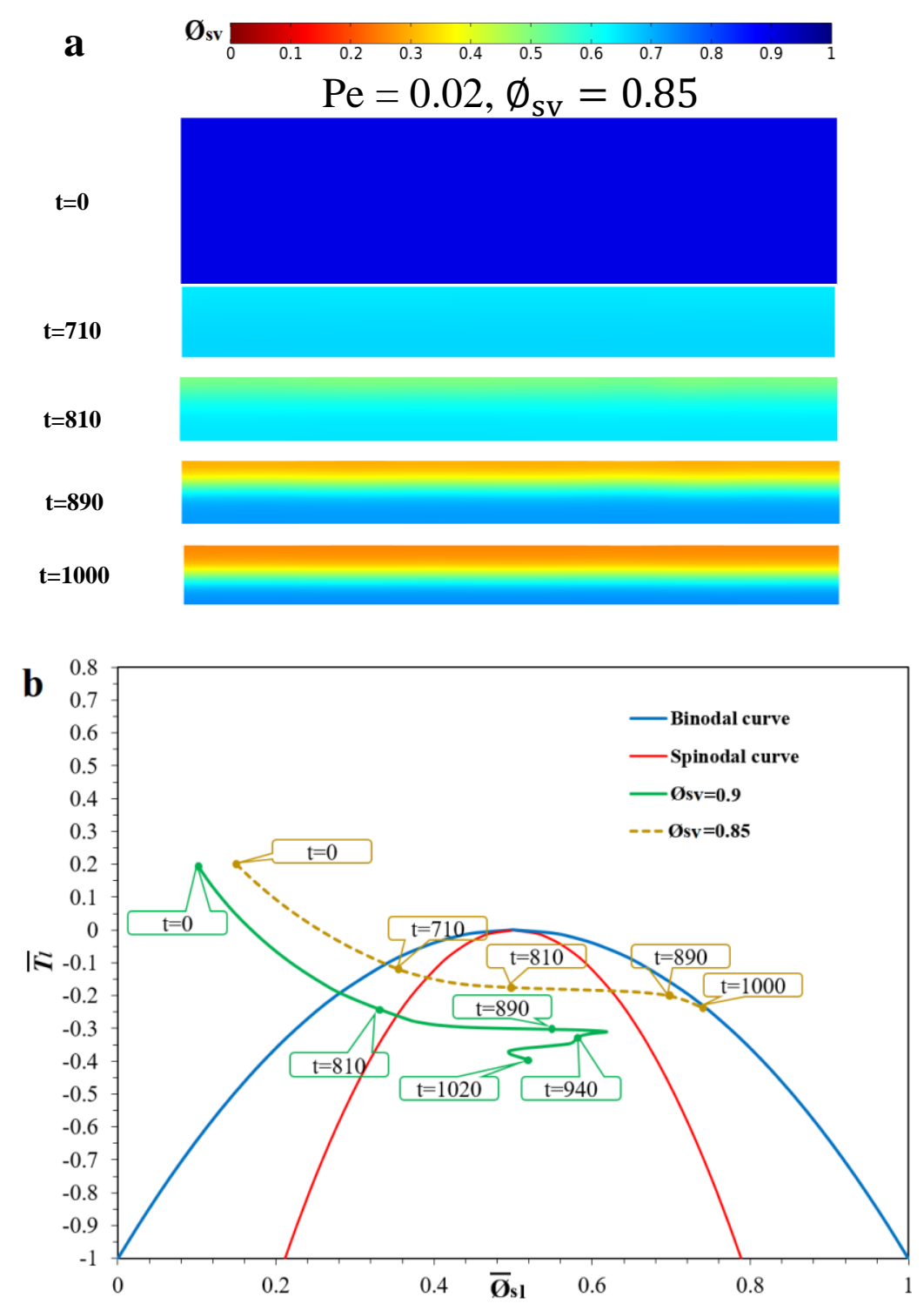

Fig. 13. a: time evolution of the phase separation morphology for initial solvent volume fraction $\emptyset_{\text {sv }}$ $=0.85$. b: path in the phase diagram describing the evolution with time of the average values of the temperature and of the solute volume faction at the liquid-gas interface for $\emptyset_{\mathrm{sv}}=0.9$ and $\emptyset_{\mathrm{sv}}=0.85$.

$$
H_{l}=20, W=80 \text {, and } T_{l}=0.2 \text { at } t=0 \text {. }
$$




\subsection{Experimental results}

In this section, we present the experimental results obtained with the Hele Shaw setup presented in subsection 2.5 and compare them with our numerical analysis. Since the distance between the plexiglass plates is small, it is assumed that convection is very weak in the system, which allows the comparison with the numerical purely diffusive approach. Note also that the experimental evaporation rate can be changed by varying the thickness $d$ of the gas layer above the thin film. In our set up, we consider $d=78 \mathrm{~mm}$ and $d=32 \mathrm{~mm}$, corresponding to "moderate" and "fast" evaporation rates, respectively. In the results presented below, the red and green colors correspond to $\mathrm{n}$-hexane-rich and DGME-rich phases, respectively. The experiment tests were performed with a liquid at the initial room temperature (between $19^{\circ} \mathrm{C}$ and $21^{\circ} \mathrm{C}$ ). This is above the critical temperature $\left(6.3^{\circ} \mathrm{C}\right.$ [87]).

For an initial mass fraction of DGME in the liquid of $8 \%$, the experimental results indicate that a moderate evaporation rate $(d=78 \mathrm{~mm})$ leads to the lateral structure (Fig. 14-a) while a fast evaporation rate $(d=32 \mathrm{~mm})$ induces the development of the skin layer (lamellar structure) at the liquid-gas interface (Fig. 14-b). This is in very good qualitative agreement with the numerical results discussed earlier.

Fig. 14-a and Fig. 14-c compare the time evolution of the phase separation for two different initial concentrations of DGME in the solution (8\% and 12\%), in the case of the moderate evaporation rate $(d=78 \mathrm{~mm})$. The lateral structure is obtained for the low initial concentration and the lamellar structure for the high initial concentration. This is again in qualitative agreement with the numerical results presented at the end of subsection 3.4. 


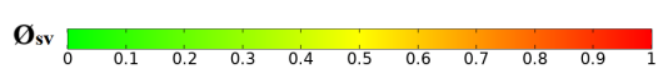

$\mathbf{a}$
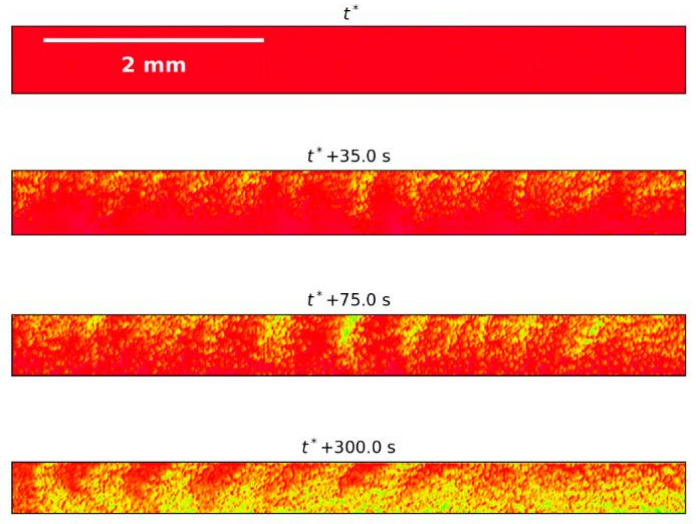

b
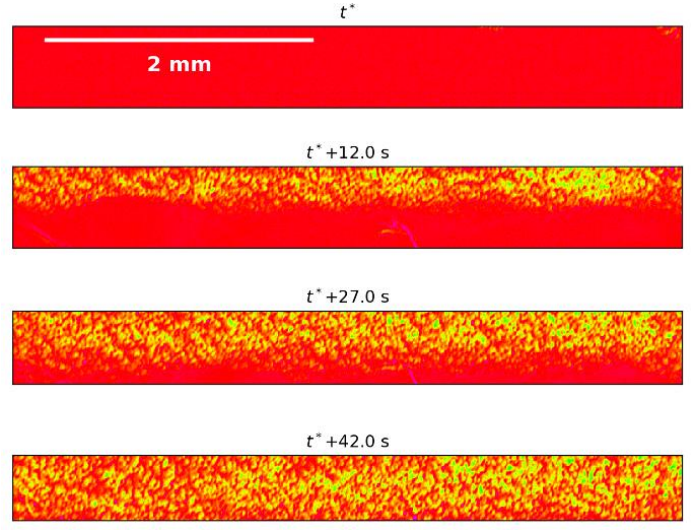

c

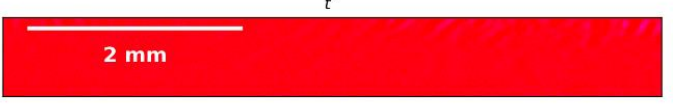

$t^{*}+12.0 \mathrm{~s}$

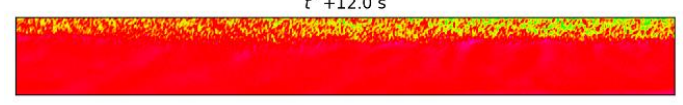

$t^{*}+20.0 \mathrm{~s}$

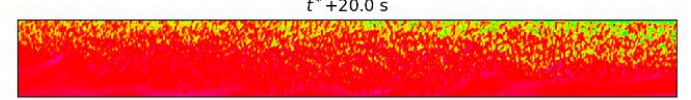

$t^{*}+36.0 \mathrm{~s}$

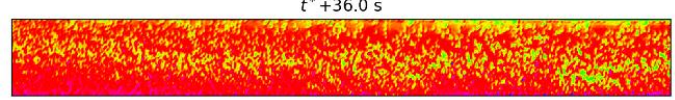

Fig. 14. Time evolution of the phase separation for: a moderate evaporation and $8 \%$ of DGME

(a), a fast evaporation and $8 \%$ of DGME (b), and a moderate evaporation and $12 \%$ of DGME (c). 


\section{Conclusion}

Thin layers of evaporating mixture of partially miscible components are commonly encountered in a number of industrial contexts. Therefore, it is of great practical interest to further understand the relationship between the physical processes that occur during the evaporation process and to analyze the morphological changes in the evaporating thin film. As mentioned in the introduction, several studies have already been published on these questions, but often ternary mixtures were considered. Such ternary systems depend on a rather large number of parameters, with the consequence that the very specific influence of one of them is not always easy to identify. In the present paper, our purpose was to consider a simpler situation, with only two components, and to try to emphasize and analyze in detail the different physical mechanisms at work when separation takes place and to determine their influence on the morphology of the separating thin film. To this end, we have developed a numerical approach, which was complemented by theoretical discussions providing important physical interpretations and by experimental work that confirmed our predictions. Our main findings about the morphology that appears in a film of a separating binary mixture are the following. First, let us mention the prominent role of the competition between evaporation and phase separation in the evolution of the system. This competition is described by the so-called Péclet number $P e$, whose value was shown to determine if a lateral or a lamellar structure is created when the phase separation begins close to the upper surface of the film. Note that for very small $P e$, the evaporation is so slow that the film remains almost uniform in the vertical direction, with the consequence that bulk phase separation occurs. This phenomenon has been studied in other works and is not described in detail in our paper. More precisely, we have shown that for moderate Péclet numbers, evaporation first creates a vertical gradient close to the interface, which subsequently brings the upper part of the film in the miscibility gap and allows a spinodal instability to take place close to the upper surface. That instability gives rise to a lateral structure and we have shown that the wavelength of the pattern decreases when $P e$ increases (i.e. when evaporation gets more efficient). This effect was related to higher wavenumbers being excited early thanks to a fast evaporation. For high values of the Péclet number, we have shown that evaporation dampens the horizontal perturbations, with the result that the spinodal instability cannot take place. Instead, phase separation occurs only in the vertical direction and a lamellar morphology is created. Interestingly, we have also shown that several physical parameters can modify the competition between evaporation and phase separation and act as kinds of regulators of the interplay between the two mechanisms. In particular, we have seen that these parameters can modify the "critical value" of the Péclet number that separates the occurrences of lateral or lamellar structures. 
Temperature, which is an easily controlled quantity, is one of these parameters and we have shown that decreasing the initial temperature of the system favors the appearance of a lateral pattern (the critical Péclet number is larger). We have also explained that this effect is linked to the entry point of the system in the miscibility gap and to the associated influence on the driving force of separation. Note that we have also indicated that the instability itself can be considered as an almost isothermal phenomenon. Then we have shown that the initial concentrations in the mixture can also modify the competition between evaporation and separation, with an increased solute concentration that favors the lamellar pattern. The mechanism of this effect is also related to the entry point in the miscibility gap. Finally, let us stress that our numerical and theoretical results regarding the lateral and lamellar structures were tested experimentally and the observations in a Hele-Shaw cell showed a very good qualitative agreement with our findings.

As far as the mid- and long-term evolution of the system is concerned, our main observations are the following. When a lateral structure is first created close to the surface, we have emphasized the possibility of coalescence of solute-rich droplets. This coalescence is observed only in thick enough layer, for which the growth of the drops can induce their coalescence before these drops reach the bottom. It is also interesting to note that more and more drops can coalesce if the thickness is larger and finally a lamellar morphology can appear in very thick films. We have also emphasized that after the creation of a lamellar structure, a spinodal instability can subsequently appear close to the substrate, as a result of the bottom part of the film crossing the spinodal curve in the phase diagram. A momentary lateral morphology, made up of solvent rich droplets, then appears close to the substrate, but this structure disappears in the long term and only solute rich fluid remains in the film. As a final general comment about the mid- and long-term evolution, it is very interesting to stress that the initial morphology that is created when phase separation starts (lamellar or lateral) can sometimes change over time and the final situation can thus be different from what the first instants could have suggested.

To finish, let us recall that our present study was restricted to the study of a binary mixture. Since lateral and lamellar morphologies are also observed in the separation of ternary mixtures, it would be very interesting to determine if the general physical mechanisms we have emphasized here are also present, with the same effects, in those situations. Remember also only a 2-D analysis was developed and that neither hydrodynamic effects, nor wetting/dewetting properties (or surface preferences) of the two components along the boundaries were considered. It is important to mention that these effects could of course play a role in the competition between the different physical mechanisms and influence the morphologies, and these effects should thus be studied in the future. 


\section{Acknowledgements}

Financial support from F.R.S.-FNRS ("DITRASOL" PDR T.0123.16) and from BELSPO ("EVAPORATION" MAP-PRODEX project) is cordially acknowledged.

\section{References}

[1] A. van Breemen, T. Zaba, V. Khikhlovskyi, J. Michels, R. Janssen, M. Kemerink, G. Gelinck, Surface Directed Phase Separation of Semiconductor Ferroelectric Polymer Blends and their Use in Non-Volatile Memories, Advanced Functional Materials, 25 (2015) 278-286.

[2] G.M. Su, E. Lim, E.J. Kramer, M.L. Chabinyc, Phase Separated Morphology of Ferroelectric-Semiconductor Polymer Blends Probed by Synchrotron X-ray Methods, Macromolecules, 48 (2015) 5861-5867.

[3] M. Haataja, F. Léonard, Influence of mobile dislocations on phase separation in binary alloys, Physical Review B, 69 (2004).

[4] P. Krasnochtchekov, R.S. Averback, P. Bellon, Homogeneous phase separation in binary alloys under ion irradiation conditions: Role of interstitial atoms, Physical Review B, 75 (2007).

[5] L. Xue, Y. Han, Pattern formation by dewetting of polymer thin film, Progress in Polymer Science, 36 (2011) 269-293.

[6] E. Kiran, K. Liu, The miscibility and phase behavior of polyethylene with poly (dimethylsiloxane) in near-critical pentane, Korean Journal of Chemical Engineering, 19 (2002) 153-158.

[7] T. Inoue, Reaction-induced phase decomposition in polymer blends, Progress in Polymer Science, 20 (1995) 119-153.

[8] L.M. Robeson, Polymer blends, A Comprehensive Review, (2007).

[9] P.K. Chan, A.D. Rey, Computational analysis of spinodal decomposition dynamics in polymer solutions, Macromolecular theory and simulations, 4 (1995) 873-899. 
[10] P.K. Chan, A.D. Rey, A numerical method for the nonlinear Cahn-Hilliard equation with nonperiodic boundary conditions, Computational materials science, 3 (1995) 377-392.

[11] K.-W.D. Lee, P.K. Chan, X. Feng, Morphology development and characterization of the phase-separated structure resulting from the thermal-induced phase separation phenomenon in polymer solutions under a temperature gradient, Chemical Engineering Science, 59 (2004) 1491-1504.

[12] J.W. Cahn, Phase Separation by Spinodal Decomposition in Isotropic Systems, The Journal of Chemical Physics, 42 (1965) 93-99.

[13] S. Asai, S. Majumdar, A. Gupta, K. Kargupta, S. Ganguly, Dynamics and pattern formation in thermally induced phase separation of polymer-solvent system, Computational Materials Science, 47 (2009) 193-205.

[14] Y.C. Li, R.P. Shi, C.P. Wang, X.J. Liu, Y. Wang, Predicting microstructures in polymer blends under two-step quench in two-dimensional space, Phys Rev E Stat Nonlin Soft Matter Phys, 83 (2011) 041502.

[15] K. Zhao, G. Zhou, Q. Wang, Y. Han, L. Wang, D. Ma, Phase Separation in Poly(9,9-dioctylfluorene)/Poly(methyl methacrylate) Blends, Macromolecular Chemistry and Physics, 211 (2010) 313-320.

[16] A. Charas, Q. Ferreira, J. Farinhas, M. Matos, L.s. Alcácer, J. Morgado, Insoluble Patterns of Cross-Linkable Conjugated Polymers from Blend Demixing in Spin Cast Films, Macromolecules, 42 (2009) 7903-7912.

[17] T.L. Tran, P.K. Chan, D. Rousseau, Morphology control in symmetric polymer blends using spinodal decomposition, Chemical Engineering Science, 60 (2005) 7153-7159.

[18] S. Puri, Kinetics of phase transitions, Kinetics of Phase Transitions, CRC Press2009, pp. 13-74.

[19] K.W.D. Lee, P.K. Chan, X. Feng, A computational study into thermally induced phase separation in polymer solutions under a temperature gradient, Macromolecular theory and simulations, 11 (2002) 996-1005. 
[20] K. Binder, P. Fratzl, Spinodal decomposition, Phase transformations in materials, (2001) 409-480.

[21] K. Binder, Surface effects on polymer blends and block copolymer melts: theoretical concepts of surface enrichment, surface induced phase separation and ordering, Acta polymerica, 46 (1995) 204-225.

[22] L.T. Yan, X.M. Xie, Wetting-layer formation mechanisms of surface-directed phase separation under different quench depths with off-critical compositions in polymer binary mixture, J Chem Phys, 126 (2007) 064908.

[23] L.-T. Yan, J. Li, Y. Li, X.-M. Xie, Kinetic pathway of pattern-directed phase separation in binary polymer mixture films, Macromolecules, 41 (2008) 3605-3612.

[24] M. Tabatabaieyazdi, P.K. Chan, J. Wu, A computational study of short-range surface-directed phase separation in polymer blends under a linear temperature gradient, Chemical Engineering Science, 137 (2015) 884-895.

[25] V.-N. Tran Duc, P.K. Chan, Using the Cahn-Hilliard Theory in Metastable Binary Solutions, ChemEngineering, 3 (2019).

[26] S. Puri, Phase separation in an off-critical quench, Physics Letters A, 134 (1988) 205-210.

[27] X. Li, M.A. Kanjwal, K. Stephansen, I.S. Chronakis, Preparing poly (caprolactone) micro-particles through solvent-induced phase separation, Materials Letters, 75 (2012) 189-191.

[28] M. Dai, L. Song, W. Nie, Y. Zhou, Golf ball-like particles fabricated by nonsolvent/solvent-induced phase separation method, Journal of Colloid and Interface Science, 391 (2013) 168-171.

[29] P.L. Evans, L.W. Schwartz, R.V. Roy, A Mathematical Model for Crater Defect Formation in a Drying Paint Layer, J Colloid Interface Sci, 227 (2000) 191-205.

[30] S. Howison, J. Moriarty, J. Ockendon, E. Terrill, S. Wilson, A mathematical model for drying paint layers, Journal of Engineering Mathematics, 32 (1997) 377394. 
[31] P. Mokarian-Tabari, M. Geoghegan, J.R. Howse, S.Y. Heriot, R.L. Thompson, R.A. Jones, Quantitative evaluation of evaporation rate during spin-coating of polymer blend films: Control of film structure through defined-atmosphere solventcasting, Eur Phys J E Soft Matter, 33 (2010) 283-289.

[32] B.-J. de Gans, U.S. Schubert, Inkjet printing of well-defined polymer dots and arrays, Langmuir, 20 (2004) 7789-7793.

[33] T. Kawase, T. Shimoda, C. Newsome, H. Sirringhaus, R.H. Friend, Inkjet printing of polymer thin film transistors, Thin Solid Films, 438-439 (2003) 279-287.

[34] B.J. de Gans, P.C. Duineveld, U.S. Schubert, Inkjet Printing of Polymers: State of the Art and Future Developments, Advanced Materials, 16 (2004) 203-213.

[35] S. Walheim, E. Schäffer, J. Mlynek, U. Steiner, Nanophase-separated polymer films as high-performance antireflection coatings, Science, 283 (1999) 520-522.

[36] A. Münch, C.P. Please, B. Wagner, Spin coating of an evaporating polymer solution, Physics of Fluids, 23 (2011).

[37] M.H. Sadafi, I. Jahn, K. Hooman, Cooling performance of solid containing water for spray assisted dry cooling towers, Energy Conversion and Management, 91 (2015) 158-167.

[38] M.H. Sadafi, J. Ruiz, M. Lucas, I. Jahn, K. Hooman, Numerical and experimental study on a single cone saline water spray in a wind tunnel, International Journal of Thermal Sciences, 120 (2017) 190-202.

[39] F.-C. Chen, Y.-K. Lin, C.-J. Ko, Submicron-scale manipulation of phase separation in organic solar cells, Applied Physics Letters, 92 (2008).

[40] K. Zhao, O. Wodo, D. Ren, H.U. Khan, M.R. Niazi, H. Hu, M. Abdelsamie, R. Li, E.Q. Li, L. Yu, B. Yan, M.M. Payne, J. Smith, J.E. Anthony, T.D. Anthopoulos, S.T. Thoroddsen, B. Ganapathysubramanian, A. Amassian, Vertical Phase Separation in Small Molecule:Polymer Blend Organic Thin Film Transistors Can Be Dynamically Controlled, Advanced Functional Materials, 26 (2016) 1737-1746.

[41] P.K. Jaiswal, S. Puri, K. Binder, Phase separation in thin films: Effect of temperature gradients, EPL (Europhysics Letters), 103 (2013). 
[42] P.K. Jaiswal, K. Binder, S. Puri, Phase separation of binary mixtures in thin films: Effects of an initial concentration gradient across the film, Phys Rev E Stat Nonlin Soft Matter Phys, 85 (2012) 041602.

[43] H. Sadafi, R. Rabani, S. Dehaeck, H. Machrafi, B. Haut, P. Dauby, P. Colinet, Evaporation induced demixing in binary sessile drops, Colloids and Surfaces A: Physicochemical and Engineering Aspects, (2020) 125052.

[44] S.Y. Heriot, R.A. Jones, An interfacial instability in a transient wetting layer leads to lateral phase separation in thin spin-cast polymer-blend films, Nat Mater, 4 (2005) 782-786.

[45] M. Sprenger, S. Walheim, A. Budkowski, U. Steiner, Hierarchic structure formation in binary and ternary polymer blends, Interface Science, 11 (2003) 225235 .

[46] S. Walheim, M. Böltau, J. Mlynek, G. Krausch, U. Steiner, Structure formation via polymer demixing in spin-cast films, Macromolecules, 30 (1997) 4995-5003.

[47] A.D. Dunbar, P. Mokarian-Tabari, A.J. Parnell, S.J. Martin, M.W. Skoda, R.A. Jones, A solution concentration dependent transition from self-stratification to lateral phase separation in spin-cast PS:d-PMMA thin films, Eur Phys J E Soft Matter, 31 (2010) 369-375.

[48] J. Jaczewska, A. Budkowski, A. Bernasik, E. Moons, J. Rysz, Polymer vs solvent diagram of film structures formed in spin-cast poly (3-alkylthiophene) blends, Macromolecules, 41 (2008) 4802-4810.

[49] G.A. Zoumpouli, S.G. Yiantsios, Hydrodynamic effects on phase separation morphologies in evaporating thin films of polymer solutions, Physics of Fluids, 28 (2016).

[50] V. Negi, O. Wodo, J.J. van Franeker, R.A.J. Janssen, P.A. Bobbert, Simulating Phase Separation during Spin Coating of a Polymer-Fullerene Blend: A Joint Computational and Experimental Investigation, ACS Applied Energy Materials, 1 (2018) 725-735. 
[51] J. Cummings, J.S. Lowengrub, B.G. Sumpter, S.M. Wise, R. Kumar, Modeling solvent evaporation during thin film formation in phase separating polymer mixtures, Soft Matter, 14 (2018) 1833-1846.

[52] P. Dayal, T. Kyu, Porous fiber formation in polymer-solvent system undergoing solvent evaporation, Journal of Applied Physics, 100 (2006).

[53] C. Schaefer, P. van der Schoot, J.J. Michels, Structuring of polymer solutions upon solvent evaporation, Physical Review E, 91 (2015).

[54] R. Ball, R. Essery, Spinodal decomposition and pattern formation near surfaces, Journal of Physics: Condensed Matter, 2 (1990) 10303.

[55] J. Kim, A numerical method for the Cahn-Hilliard equation with a variable mobility, Communications in Nonlinear Science and Numerical Simulation, 12 (2007) 1560-1571.

[56] M. Dehghan, V. Mohammadi, The numerical solution of Cahn-Hilliard $(\mathrm{CH})$ equation in one, two and three-dimensions via globally radial basis functions (GRBFs) and RBFs-differential quadrature (RBFs-DQ) methods, Engineering Analysis with Boundary Elements, 51 (2015) 74-100.

[57] J.F. Blowey, C.M. Elliott, The Cahn-Hilliard gradient theory for phase separation with non-smooth free energy Part I: Mathematical analysis, European Journal of Applied Mathematics, 2 (2009) 233-280.

[58] J.W. Cahn, J.E. Hilliard, Free Energy of a Nonuniform System. I. Interfacial Free Energy, The Journal of Chemical Physics, 28 (1958) 258-267.

[59] C. Zhou, P. Yue, J.J. Feng, C.F. Ollivier-Gooch, H.H. Hu, 3D phase-field simulations of interfacial dynamics in Newtonian and viscoelastic fluids, Journal of Computational Physics, 229 (2010) 498-511.

[60] P. Yue, C. Zhou, J.J. Feng, C.F. Ollivier-Gooch, H.H. Hu, Phase-field simulations of interfacial dynamics in viscoelastic fluids using finite elements with adaptive meshing, Journal of Computational Physics, 219 (2006) 47-67.

[61] A. Bertei, R. Mauri, Dynamics of phase separation of sheared inertialess binary mixtures, Physics of Fluids, 32 (2020). 
[62] A. Das, S.M. Ali, Understanding of interfacial tension and interface thickness of liquid/liquid interface at a finite concentration of alkyl phosphate by molecular dynamics simulation, Journal of Molecular Liquids, 277 (2019) 217-232.

[63] D.M. Saylor, C.S. Kim, D.V. Patwardhan, J.A. Warren, Diffuse-interface theory for structure formation and release behavior in controlled drug release systems, Acta Biomater, 3 (2007) 851-864.

[64] T. Araki, H. Tanaka, Hydrodynamic delocalization of phase separation in a locally cooled fluid mixture, Europhysics Letters (EPL), 65 (2004) 214-220.

[65] O. Wodo, B. Ganapathysubramanian, Computationally efficient solution to the Cahn-Hilliard equation: Adaptive implicit time schemes, mesh sensitivity analysis and the 3D isoperimetric problem, Journal of Computational Physics, 230 (2011) 6037-6060.

[66] H. Cook, Brownian motion in spinodal decomposition, Acta metallurgica, 18 (1970) 297-306.

[67] J.S. Langer, Theory of spinodal decomposition in alloys, Annals of Physics, 65 (1971) 53-86.

[68] A. Bertei, B. Tellini, R. Mauri, Dynamic transition of dendrite orientation in the diffusive spinodal decomposition of binary mixtures under a thermal gradient, Chemical Engineering Science, 203 (2019) 450-463.

[69] R. Rabani, H. Machrafi, P. Dauby, Effect of including a gas layer on the gel formation process during the drying of a polymer solution, Eur Phys J E Soft Matter, 40 (2017) 89.

[70] R. Rabani, H. Machrafi, P. Dauby, Influence of Composition Dependent Diffusion Coefficient, Viscosity and Relaxation Time on Evaporative RayleighBénard-Marangoni Instabilities Induced by Solvent Evaporation in a Polymer Solution, Microgravity Science and Technology, 31 (2019) 615-628.

[71] P. Colinet, J.C. Legros, M.G. Velarde, Nonlinear dynamics of surface-tensiondriven instabilities, Wiley-vch2001. 
[72] M.G. Hennessy, G.L. Ferretti, J.T. Cabral, O.K. Matar, A minimal model for solvent evaporation and absorption in thin films, J Colloid Interface Sci, 488 (2017) 61-71.

[73] K.y. Ozawa, T. Okuzono, M. Doi, Diffusion Process during Drying to Cause the Skin Formation in Polymer Solutions, Japanese Journal of Applied Physics, 45 (2006) 8817-8822.

[74] O. Wodo, B. Ganapathysubramanian, Modeling morphology evolution during solvent-based fabrication of organic solar cells, Computational Materials Science, 55 (2012) 113-126.

[75] C.S. Kim, D.M. Saylor, M.K. McDermott, D.V. Patwardhan, J.A. Warren, Modeling solvent evaporation during the manufacture of controlled drug-release coatings and the impact on release kinetics, J Biomed Mater Res B Appl Biomater, 90 (2009) 688-699.

[76] COMSOL Multiphysics, User's Guide Version 5.5, (2019).

[77] C.M. Elliott, D.A. French, F. Milner, A second order splitting method for the Cahn-Hilliard equation, Numerische Mathematik, 54 (1989) 575-590.

[78] C.M. Elliott, H. Garcke, On the Cahn-Hilliard equation with degenerate mobility, Siam journal on mathematical analysis, 27 (1996) 404-423.

[79] S. Zhang, M. Wang, A nonconforming finite element method for the CahnHilliard equation, Journal of Computational Physics, 229 (2010) 7361-7372.

[80] P. Yazgan-Birgi, H.A. Arafat, M.I. Hassan Ali, Implementation of two multiphase flow methods in modeling wetting of microporous hydrophobic membranes, Sci Total Environ, 691 (2019) 1251-1261.

[81] M.A. Zaeem, S.D. Mesarovic, Morphological instabilities in thin films: Evolution maps, Computational Materials Science, 50 (2011) 1030-1036.

[82] M. Krivilyov, D. Aflyatunova, V. Lebedev, P.K. Galenko, Phase-field simulation of non-isothermal phase separation in rapidly quenched $\mathrm{Co}-\mathrm{Cu}$ melts, Computational Materials Science, 158 (2019) 289-295. 
[83] M. Liang, X. Xin, W. Fan, H. Wang, W. Sun, Phase field simulation and microscopic observation of phase separation and thermal stability of polymer modified asphalt, Construction and Building Materials, 204 (2019) 132-143.

[84] G. Fayaz, S. Kazemzadeh, Towards additive manufacturing of compressor impellers: 3D modeling of multilayer laser solid freeform fabrication of nickel alloy 625 powder mixed with nano-CeO 2 on AISI 4140, Additive Manufacturing, 20 (2018) 182-188.

[85] A. Adrover, A. Brasiello, G. Ponso, A moving boundary model for food isothermal drying and shrinkage: General setting, Journal of Food Engineering, 244 (2019) 178-191.

[86] M.-A. Xue, O. Kargbo, J. Zheng, Seiche oscillations of layered fluids in a closed rectangular tank with wave damping mechanism, Ocean Engineering, 196 (2020).

[87] R. Martínez, J.A. Gonzalez, I. Garcia de la Fuente, J.C. Cobos, Thermodynamic properties of n-alkoxyethanols+ organic solvent mixtures. XIV. Liquid- liquid equilibria of systems containing 2-(2-ethoxyethoxy) ethanol and selected alkanes, Journal of Chemical \& Engineering Data, 45 (2000) 1036-1039.

[88] X. Li, G. Ji, H. Zhang, Phase transitions of macromolecular microsphere composite hydrogels based on the stochastic Cahn-Hilliard equation, Journal of Computational Physics, 283 (2015) 81-97.

[89] Y.C. Li, R.P. Shi, C.P. Wang, X.J. Liu, Y. Wang, Phase-field simulation of thermally induced spinodal decomposition in polymer blends, Modelling and Simulation in Materials Science and Engineering, 20 (2012).

[90] S.S. Kim, T.H. Sanders, Phase-field simulation of spinodal phase separation in the Na2O-SiO2 glasses, Journal of Non-Crystalline Solids, 528 (2020).

[91] Y. Li, Thermodynamic and kinetic study of spinodal phase separation in heptane-phenol system, Calphad, 50 (2015) 113-117.

[92] O. Wodo, B. Ganapathysubramanian, How do evaporating thin films evolve? Unravelling phase-separation mechanisms during solvent-based fabrication of polymer blends, Applied Physics Letters, 105 (2014) 153104. 
[93] K. Binder, Spinodal Decomposition in P. Haasen (Ed.): Material Science and Technology, Vol. 5, Phase Transitions in Materials, (1991).

[94] R. Rabani, Some Effects of Evaporation in Binary Mixtures, Université de Liège, Liege, Belgium, 2020.

[95] P. Papon, J. Leblond, P.H. Meijer, Physics of Phase Transitions, Springer2002.

[96] H. Machrafi, A. Rednikov, P. Colinet, P.C. Dauby, Time-dependent Marangoni-Bénard instability of an evaporating binary-liquid layer including gas transients, Physics of Fluids, 25 (2013) 084106.

[97] H. Machrafi, A. Rednikov, P. Colinet, P. Dauby, Bénard instabilities in a binary-liquid layer evaporating into an inert gas, Journal of colloid and interface science, 349 (2010) 331-353. 